\title{
Stationary, Axisymmetric Neutron Stars with Meridional Circulation in General Relativity
}

\author{
Reiner Birkl ${ }^{1}$, Nikolaos Stergioulas ${ }^{2}$, Ewald Müller ${ }^{1}$ \\ ${ }^{1}$ Max-Planck-Institut für Astrophysik, 85741 Garching, Germany \\ ${ }^{2}$ Aristotle University of Thessaloniki, 54124 Thessaloniki, Greece
}

\begin{abstract}
We present the first stationary, axisymmetric neutron star models with meridional circulation in general relativity. For that purpose, we developed GRNS, a new code based on a fixed point iteration. We find a two-dimensional set of meridional circulation modes, which differ by the number of vortices in the stream lines of the neutron star fluid. For expected maximal meridional circulation velocities of about $1000 \mathrm{~km} / \mathrm{s}$, the vortices cause surface deformations of about a percent. The deformations depend on the shape of the vortices close to the surface and increase with the meridional circulation velocity. We also computed models of rotating neutron stars with meridional circulation, where neither the surface rotates nor does the rotation velocity exceed the circulation velocity.
\end{abstract}

PACS numbers: 97.60.Jd, 04.20.-q, 95.30.Lz

\section{INTRODUCTION}

The shape of a neutron star depends on the motion of the stellar fluid. This motion can be decomposed into two components with respect to the rotation axis, which contains the center of mass and points in the direction of the total angular momentum. The first component is differential rotation around the rotation axis. This kind of motion, which leads to a flattening of the neutron star surface, has been investigated intensively, also in general relativity $[1,2]$. The second component is meridional circulation, where the flow occurs in meridional planes, i.e. planes containing the rotation axis. Its influence, which may play a role for new-born neutron stars, is not yet well understood. Earlier investigations involve severe limitations, as they were either restricted to the Newtonian framework [3] or they were of perturbative nature only [4]. We are interested in how meridional circulations of arbitrary strength may influence the shape of a general relativistic neutron star. Moreover, contrary to the earlier studies considering non-perturbative fluid motions, where in each case only one of the two kinds of motions was considered alone, we also investigate the combined effect of differential rotation and meridional circulation.

For that purpose, we developed a new program, called GRNS (=Generally Rotating Neutron Star), as the complexity of Einstein's field equation makes a numerical approach to our investigation unavoidable. GRNS combines the methods applied in two programs created by two of us more than a decade ago. The first one is RNS [1,2] , a general relativistic code for computing models of rapidly rotating neutron stars. This code is based on a fixed point iteration, and the theoretical considerations of Komatsu et al. [5]. However, RNS is limited to differentially rotating configurations and does not allow for meridional circulation. The situation is just opposite for the second program (E. Müller [3]), which computes meridional circulation in non-rotating Newtonian configurations. It uses a Newton-Raphson iteration scheme to solve for the stationary equilibrium configurations, and describes the flow by means of a scalar function, the so-called stream function. Below we will show how the stream function method can be extended to general relativity. We do not use the approach of Komatsu et al. [5] to describe the effects of meridional circulation on the curvature of spacetime, but rely on the more general, theoretical investigation of Gourgoulhon et al. [6], who formulated Einstein's field equation as a set of covariant Poisson equations. The latter can be rewritten as Poisson equations in flat space such that Green functions can be used to solve them. We have incorporated these theoretical extensions into GRNS, including the fixed point iteration of RNS, and a modified Tolman-Oppenheimer-Volkoff solution as the initial guess. That way, GRNS is able to handle differentially rotating neutron stars with meridional circulation.

GRNS uses a simplified neutron star model. Considering the neutron star during a sufficiently short time interval after its formation, it is an appropriate approximation to assume stationarity. In addition, we limit ourselves to axisymmetric configurations. For simplicity, the stellar matter in our simulations is assumed to be a perfect fluid described by a barotropic equation of state, although the theoretical formulation presented in this paper is kept general enough to deal with arbitrary equations of state. We further assume that the neutron star has no substructure (i.e., crust, core, etc.), and that its chemical composition is homogeneous. Finally, we neglect the influence of magnetic fields.

The paper is organized as follows. In Sec. II, we rewrite the covariant Poisson equations of Gourgoulhon et al. [6] to flat-space Poisson equations and extend the Newtonian stream function method [3] to general relativity. Intermediate steps during these computations are listed in the Appendix. Sec. III gives a closer look at the GRNS code explaining the fixed point iteration scheme and showing how the flat-space Poisson equations can be solved by means of Green functions. Actual neutron star models are presented in Sec. IV, and we conclude our paper in Sec. V. 


\section{THEORY}

\section{A. Notations and conventions}

We use geometrized units for the theoretical computations, where the speed of light $c$ and the gravitational constant $G$ are set equal to unity. The results obtained from neutron star simulations are presented in the more appropriate cgs units. The general relativistic formalism adopts Einstein's sum convention and is kept as close as possible to the conventions chosen in Gourgoulhon et al. [6]. However, we make a slightly different choice for the range of tensor indices. In our specific choice of coordinates, concrete indices for 4-tensors, 3-tensors and 2-tensors are used as follows:

$$
\begin{aligned}
& \alpha, \beta, \ldots \in\{t, r, \theta, \phi\}, \\
& a, b, \ldots \in\{r, \theta, \phi\}, \\
& m, n, \ldots \in\{r, \theta\},
\end{aligned}
$$

with the time $t$, the radius $r$, and the angles $\theta$ and $\phi$. Following Misner et al. [7], we choose $(-,+,+,+)$ as the signature of the metric.

\section{B. Basic fields}

We begin our theoretical considerations by defining a set of physical fields, the so-called basic fields, which determine the state of the neutron star uniquely. Due to the assumption of stationarity and axisymmetry, these and all other fields depend on only two coordinates, the radius $r$ and the polar angle $\theta$. There are two types of basic fields. The first set describes the curvature of spacetime, and its fields are denoted basic geometry fields. The second set are hydrodynamic fields, called basic matter fields, which specify the properties of the stellar fluid. In the following, we will define the basic geometry fields without giving an explanation of how these fields come into being. For that purpose, we refer to Gourgoulhon et al. [6].

The curvature of spacetime is described by the metric $g_{\alpha \beta}$. Using a $(2+1)+1$ decomposition of spacetime, the ten independent components of the 4-metric $g_{\alpha \beta}$ are rewritten to new fields in Gourgoulhon et al. [6]. These fields consist of two scalars fields, the 3-lapse $N$ and the 2-lapse $M$, two vector fields, the 3 -shift $N^{a}$ and the 2shift $M^{m}$, and the 2 -metric $k_{m n}$, respectively:

$$
\left(\begin{array}{cc}
g_{t t} & g_{t b} \\
g_{a t} & g_{a b}
\end{array}\right)=\left(\begin{array}{cc}
N_{c} N^{c}-N^{2} & -N_{b} \\
-N_{a} & h_{a b}
\end{array}\right),
$$

with the 3 -metric

$$
\left(\begin{array}{cc}
h_{m n} & h_{m \phi} \\
h_{\phi n} & h_{\phi \phi}
\end{array}\right)=\left(\begin{array}{cc}
k_{m n} & -M_{m} \\
-M_{n} & M^{2}+M_{p} M^{p}
\end{array}\right)
$$

and

$$
\begin{aligned}
N_{a} & =h_{a b} N^{b} \\
M_{m} & =k_{m n} M^{n} .
\end{aligned}
$$

Using a special choice of coordinates, which are denoted as MTCMA (maximal time slicing - conformally minimal azimuthal slicing) in Gourgoulhon et al. [6], these authors write the 2-metric $k_{m n}$ such that the only non-vanishing components are $k_{r r}=A^{2}$ and $k_{\theta \theta}=A^{2} r^{2}$, where $A$ shall be called the 2-conformal factor. Adopting the definitions $[6]$

$$
\begin{aligned}
\nu & =\ln N, \\
\alpha & =\ln A, \\
\beta & =\ln \frac{M}{r \sin \theta},
\end{aligned}
$$

we then call the eight functions

$$
\nu, \alpha, \beta, M^{r}, M^{\theta}, N^{r}, N^{\theta}, N^{\phi}
$$

basic geometry fields. In contrast, for circular spacetimes (i.e. without meridional circulation), only four geometry fields are required.

The neutron star fluid is described by a stress-energy tensor

$$
T_{\alpha \beta}=(\epsilon+p) u_{\alpha} u_{\beta}+p g_{\alpha \beta},
$$

with the total energy density $\epsilon$, pressure $p$, and 4-velocity $u^{\alpha}$. Due to the constraint

$$
g_{\alpha \beta} u^{\alpha} u^{\beta}=-1
$$

the 4-velocity $u^{\alpha}$ possesses only three independent degrees of freedom. One of them is the specific angular momentum, i.e. the $\phi$-component of

$$
l_{a}=-\frac{u_{a}}{u_{t}}
$$

Under our symmetry assumptions (Sect. II E), the other two degrees of freedom can be expressed in terms of a stream function $\psi$, an approach known to be working fine in Newtonian gravity (equations (2) and (3) in Eriguchi et al. [3]). Further below (Sect. II E), we will demonstrate that this approach holds in general relativity, too. There, we will also explain how the 4 -velocity $u^{\alpha}$ can be obtained from the stream function $\psi$ and the specific angular momentum $l_{\phi}$. However, by convention we do not call the stream function $\psi$ a basic field, but the modified stream function [3]

$$
\chi_{0}=\frac{\psi}{r \sin \theta}
$$

So, we refer to

$$
\epsilon, p, \chi_{0}, l_{\phi}
$$

as basic matter fields. Actually, we have chosen the twelve basic fields (5) and (10) in such a manner that they vanish for an empty Minkowski spacetime, which is advantageous for our numerical method. 


\section{Geometry equations}

Each of the basic fields (5) and (10) is specified by a field equation. In this section, we will address the basic geometry fields, which are governed by Einstein's field equation

$$
G_{\alpha \beta}=8 \pi T_{\alpha \beta},
$$

where $G_{\alpha \beta}$ is the Einstein tensor. Unfortunately, this equation is written in such a compact manner that it is practically impossible to directly solve for the basic geometry fields (5). Therefore, we use the theoretical consideration of Gourgoulhon et al. [6], who rewrite equation (11) as a set of eight covariant Poisson equations, each of the form

$$
\Delta_{\mathrm{cov}} \Phi=S
$$

where $\Delta_{\text {cov }}, \Phi$, and $S$ are a covariant Laplacian, a potential, and a source, respectively. In that paper, the covariant Poisson equations are labelled (B3-B7). Trying to validate these equations, we found that the three equations (B3), (B4a) and (B4b) contain minor errors. In Appendix A, we show the corrected versions of these equations.

Let us now have a closer look at the covariant Laplacian $\Delta_{\text {cov }}$ appearing in equation (12). This operator is the scalar product of two covariant derivatives. Such derivatives can be split into a partial derivative and corrections arising from the curvature of spacetime, expressed in terms of the connection coefficients. In a similar manner, it is possible to express a covariant Laplacian $\Delta_{\text {cov }}$ in terms of the corresponding flat-space Laplacian $\Delta_{\text {flat }}$ and curvature corrections. That way, equation (12) can be rewritten as

$$
\Delta_{\text {flat }} \Phi=S^{\prime}
$$

with some new source $S^{\prime}$. In this way, we arrive at the following flat-space versions of equations (B3-B7) in [6]:

$$
\begin{aligned}
{ }^{3} \Delta \nu & =S_{\nu}, \\
{ }^{3} \Delta^{a}{ }_{b} N^{b} & =S_{N}^{a}, \\
{ }^{2} \Delta[r \sin \theta(\beta+\nu)] & =S_{\beta}, \\
{ }^{2} \Delta^{m}{ }_{n}\left[e^{2(\alpha+\nu)} M^{n}\right] & =S_{M}^{m}, \\
{ }^{2} \Delta(\alpha+\nu) & =S_{\alpha} .
\end{aligned}
$$

The flat-space Laplacians ${ }^{2} \Delta,{ }^{3} \Delta,{ }^{2} \Delta^{m}{ }_{n}$ and ${ }^{3} \Delta^{a}{ }_{b}$ are listed in Appendix B, and the rather lengthy sources $S_{\nu}$, $S_{\alpha}, S_{\beta}, S_{M}^{m}$ and $S_{N}^{a}$ in Appendix I, respectively. Modifying the sources $S_{\beta}, S_{M}^{m}$, and $S_{\alpha}$ properly, it is possible to rewrite equations (15-17) such that the Laplacians in these equations act directly on the basic geometry fields $\beta, M^{m}$, and $\alpha$, but not on expressions containing these fields. However, the numerical stability of the fixed point iteration is highly sensitive to such changes, and in that case the iteration would diverge. By experimenting with the GRNS code, we found that the choice made in equations (15-17) produces convergent results.

For rotating neutron stars, we achieved convergence only by setting the source $S_{M}^{m}$ equal to zero on the grid lines adjacent to the rotation axis. Even when increasing the grid resolution, only the adjacent grid lines have to be modified, and thus setting the source equal to zero is a valid procedure.

\section{Matter equations}

The basic matter fields (10) are fixed by the equation of state and the equation of general relativistic hydrodynamics

$$
\nabla_{\beta} T^{\alpha \beta}=0
$$

where $\nabla_{\alpha}$ is the covariant derivative. As will become apparent later (Sect. II G), for a stationary configuration, the equations of hydrodynamics are integrable under the assumption of a barotropic fluid, where the total energy density $\epsilon$ is a function $\epsilon(p)$ of the pressure $p$ only.

Similar to Einstein's field equation (11), equation (18) is again too compact to be solved directly. To overcome this problem, we introduce the projector

$$
q_{\beta}^{\alpha}=\delta_{\beta}^{\alpha}+u^{\alpha} u_{\beta}
$$

orthogonal to the 4 -velocity $u^{\alpha}$, where $\delta_{\beta}^{\alpha}$ is the Kronecker symbol. Then, we project equation (18) in the direction parallel and orthogonal to the 4-velocity:

$$
\begin{aligned}
& u_{\alpha} \nabla_{\beta} T^{\alpha \beta}=0, \\
& q_{\alpha}^{\gamma} \nabla_{\beta} T^{\alpha \beta}=0,
\end{aligned}
$$

and bring these two equations in the usual form

$$
\begin{aligned}
\nabla_{\alpha}\left[(\epsilon+p) u^{\alpha}\right] & =u^{\alpha} \nabla_{\alpha} p, \\
(\epsilon+p) u^{\beta} \nabla_{\beta} u^{\alpha} & =-q^{\alpha \beta} \nabla_{\beta} p,
\end{aligned}
$$

which are the energy equation and the general relativistic Euler equation. In the subsequent sections we will extend the Newtonian stream function method [3] to general relativity by a further reformulation of equations (19) and (20).

\section{E. Stream function}

The stream function $\psi$ is introduced to solve the energy equation (19). In Appendix C, we show that due to our symmetry assumptions equation (19) can be written as the vanishing flat-space 3-divergence

$$
\partial_{m} j^{m}+\frac{2}{r} j^{r}+\cot \theta j^{\theta}=0
$$

of the 3 -vector

$$
j^{a}=\varrho u^{a}
$$


with [5]

$$
\begin{aligned}
& \varrho=A^{2} e^{\gamma}(\epsilon+p) u_{t}, \\
& \gamma=\beta+\nu
\end{aligned}
$$

(note that the symbol $\varrho$ is different than the rest mass density $\rho$ introduced later). Then, similar to the Newtonian stream function method [3], equation (21) is automatically satisfied by the relation

$$
\left(\begin{array}{c}
u^{r} \\
u^{\theta}
\end{array}\right)=\frac{1}{\varrho r^{2} \sin \theta}\left(\begin{array}{c}
\partial_{\theta} \psi \\
-\partial_{r} \psi
\end{array}\right) .
$$

This relation reduces two degrees of freedom of the 4velocity $u^{\alpha}$ to the one of the stream function $\psi$ (which has the units $[g / s])$. In the Newtonian limit, where $u_{t}=-1$, these two degrees of freedom correspond to the two components $u^{m}$. Unfortunately, in general relativity, the situation is somewhat more complicated, because the components $u^{m}$ together with the third degree of freedom $u^{\phi}$ also appear on the right hand side of equation (24), hidden in the quantity $u_{t}$. However, using equation (24), the constraint (7), and the definition (8) it is straightforward to show that the 4 -velocity $u^{\alpha}$ can still be computed from the stream function $\psi$ and the specific angular momen$\operatorname{tum} l_{\phi}$.

\section{F. Specific angular momentum}

Taking stationarity and axisymmetry into account, a short calculation shows that the general relativistic Euler equation (20) can be written as

$$
\frac{\partial_{\alpha} p+u_{\alpha} u^{m} \partial_{m} p}{\epsilon+p}=\frac{1}{2} u^{\beta} u^{\gamma} \partial_{\alpha} g_{\beta \gamma}-u^{m} \partial_{m} u_{\alpha}
$$

We recall that this equation is a projection orthogonal to the 4-velocity $u^{\alpha}$ and has thus only three independent degrees of freedom. In the following, we will therefore consider only the three spatial components of equation (25) by setting $\alpha=a$ in that equation. However, we use the temporal component $\alpha=t$, shown in equation (C3), to rewrite these spatial components as

$$
\frac{\partial_{a} p}{\epsilon+p}=\frac{1}{2} u^{\beta} u^{\gamma} \partial_{a} g_{\beta \gamma}+u_{t} u^{m} \partial_{m} l_{a} .
$$

Let us start with the azimuthal component of equation (26). For that purpose, we set $a=\phi$ and being aware that all azimuthal derivatives $\partial_{\phi} \ldots$ vanish due to axisymmetry, we obtain

$$
u^{m} \partial_{m} l_{\phi}=0
$$

which extends equation (12) of Eriguchi et al. [3] to general relativity. To solve equation (27), we discern the three cases
(A) $u^{m}=0$ everywhere,
(B) $u^{m} \neq 0$ somewhere,
(C) $u^{m} \neq 0$ everywhere.

In case (A), for which equation (27) is satisfied automatically, the neutron star can be differentially rotating, but without any meridional circulation. We are not interested in this case, because it has already been investigated with the RNS code $[1,2,8]$. Case (B) allows a meridional circulation, but not everywhere in the neutron star. In this paper, we do not investigate such solutions nor do we analyze whether they exist at all. Instead, we focus on case $(\mathrm{C})$, where meridional circulation is present everywhere in the star. For that case, we use equation (24) and rewrite equation (27) as

$$
\partial_{\theta} \psi \partial_{r} l_{\phi}-\partial_{r} \psi \partial_{\theta} l_{\phi}=0
$$

This equation is satisfied by

$$
l_{\phi}=L(\psi) \text {, }
$$

where $L(\psi)$ is an arbitrary function of the stream function $\psi[9]$.

\section{G. Poisson equation for modified stream function}

We proceed with the meridional components of equation (26), which can be written as

$$
\frac{\partial_{m} p}{\epsilon+p}+\frac{1}{2} \partial_{m} \ln \left(u_{t}\right)^{2}=r w u_{t}\left(\begin{array}{c}
u^{\theta} \\
-u^{r}
\end{array}\right)-u_{t} u^{\phi} \partial_{m} l_{\phi}
$$

according to Appendix D, where the quantity $w$ is defined in equation (D2). This expression extends equations (7) and (8) of Eriguchi et al. [3] to general relativity. Our limitation to a barotropic equation of state allows us to write the left hand side of equation (29) in the form of a gradient. To this end, we introduce the heat function [10]

$$
H(p)=\int_{0}^{p} \frac{\mathrm{d} p^{\prime}}{\epsilon\left(p^{\prime}\right)+p^{\prime}} .
$$

For the right hand side, we use equations (24) and (28). Then, equation (29) reads

$$
\begin{aligned}
& \partial_{m}\left[H(p)+\frac{1}{2} \ln \left(u_{t}\right)^{2}\right] \\
= & -u_{t}\left(\frac{w}{\varrho r \sin \theta}+u^{\phi} L^{\prime}(\psi)\right) \partial_{m} \psi,
\end{aligned}
$$

which implies that the right hand side must be a gradient, too, for obtaining a first integral of motion. This leads to the condition

$$
w=\varrho r \sin \theta\left(\frac{f(\psi)}{u_{t}}-u^{\phi} L^{\prime}(\psi)\right),
$$

with an arbitrary function $f(\psi)$. Examining equations (D2), (8), and eventually (24), we realize that the quantity $w$ contains second derivatives of the stream function $\psi$. Actually, as shown in the Appendix E, it turns out 
that there is a Laplacian hidden in that quantity, and that condition (32) is equivalent to

$$
\frac{1}{\cos \phi}{ }^{3} \Delta\left(\chi_{0} \cos \phi\right)=S_{\chi_{0}},
$$

where the modified stream function $\chi_{0}(9)$ is used, and the source $S_{\chi_{0}}$ is given in Appendix I. This flat-space Poisson equation differs from that presented in Sect. II C by the appearance of the angle $\phi$.

\section{H. Pressure}

We now return to equation (31) and insert condition (32). Then, by integration we obtain

$$
\frac{1}{2} \ln \left(u_{t}\right)^{2}+H(p)+I(\psi)=C,
$$

with the ancillary function

$$
I(\psi)=\int_{0}^{\psi} \mathrm{d} \psi^{\prime} f\left(\psi^{\prime}\right)
$$

and the integration constant $C$. The heat function $H(p)$ appearing in equation (34) is invertible, because the total energy density $\epsilon$ and the pressure $p$ are both positive quantities, and thus also the integrand of equation (30), such that the heat function $H(p)$ is strictly monotonous. Denoting the inverse heat function as $H^{-1}(p)$, equation (34) the pressure can be expressed as

$$
p=H^{-1}\left(H\left(p_{\mathrm{c}}\right)+\frac{1}{2} \ln \left(\frac{u_{t}^{\mathrm{c}}}{u_{t}}\right)^{2}+I\left(\psi_{\mathrm{c}}\right)-I(\psi)\right),
$$

with the central pressure $p_{\mathrm{c}}$, the central stream function $\psi_{\mathrm{c}}$, and the central covariant temporal component $u_{t}^{\mathrm{c}}$ of the 4 -velocity, respectively. In general, the above inversion is performed numerically.

\section{NUMERICS}

\section{A. Fixed point iteration}

In Sect. II, we have introduced the basic fields

$$
\nu, \alpha, \beta, M^{r}, M^{\theta}, N^{r}, N^{\theta}, N^{\phi}, p, \epsilon, \chi_{0}, l_{\phi}
$$

and their governing equations (13-17), (28), (33), (35) and the equation of state. We solve these equations by means of a fixed-point iteration after having specified an initial guess $\nu_{0}, \ldots, l_{\phi, 0}$, which is constructed in the following way. We set the basic fields (36) to the Tolman-Oppenheimer-Volkoff solution. This spherically symmetric solution has no meridional circulation, and therefore the basic matter field $\chi_{0}$ vanishes. Then, we modify the Tolman-Oppenheimer-Volkoff solution such

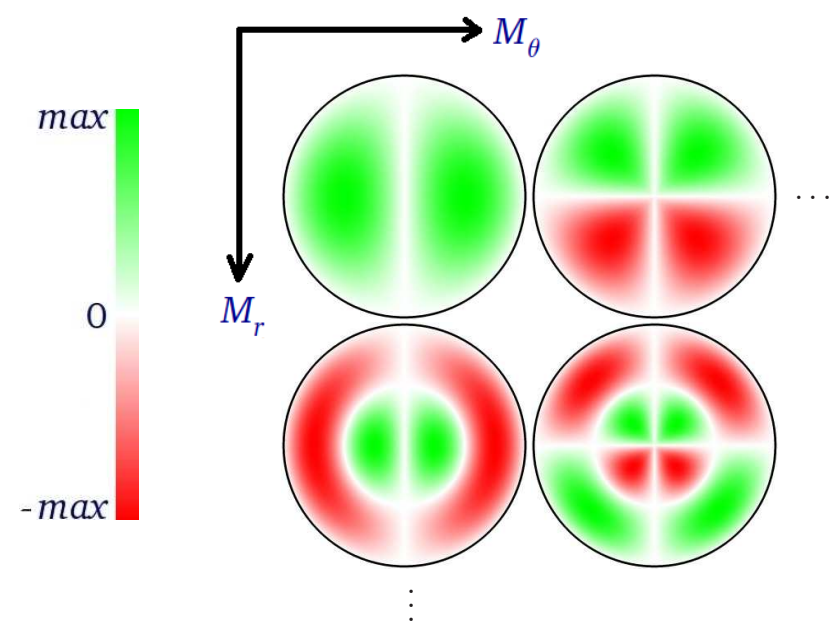

FIG. 1: Initial guess of basic field $\chi_{0}$. Each one of the four panels shows the distribution of the field $\chi_{0}$ inside of the neutron star for one choice of the pair $\left(\mathcal{M}_{r}, \mathcal{M}_{\theta}\right)$ according to equation (38). The top, left panel visualizes the case $\left(\mathcal{M}_{r}, \mathcal{M}_{\theta}\right)=(1,1)$. Proceeding to the right increases the value of the parameter $\mathcal{M}_{\theta}$, and we have to go down to raise the value of $\mathcal{M}_{r}$. For each panel, the maximal absolute field value is called $\max$. The values $\max$ and $-\max$ are represented by the brightest red and green colors, respectively.

that the field $\chi_{0}$ becomes non-zero. For that purpose, we consider surface-adapted coordinates

$$
\begin{aligned}
\tilde{r} & =\frac{r}{R(\theta)}, \\
\tilde{\theta} & =\theta,
\end{aligned}
$$

where $R(\theta)$ is the radial coordinate of the neutron star surface. Then, the invariance of scalars tells us

$$
\tilde{\chi}_{0}(\tilde{r}, \tilde{\theta})=\chi_{0}(r, \theta)
$$

and we set

$$
\tilde{\chi}_{0}(\tilde{r}, \tilde{\theta})=\chi_{0}^{\max } \sin \left(\mathcal{M}_{r} \tilde{r} \pi\right) \sin \left(\mathcal{M}_{\theta} \tilde{\theta}\right),
$$

with an arbitrary constant $\chi_{0}^{\max }$ and parameters $\mathcal{M}_{r}, \mathcal{M}_{\theta} \in\{1,2, \ldots\}$, as shown in Fig. 1 .

To describe the iteration steps, we denote the values of the basic fields (36) after the $s$-th iteration step by $\nu_{s}, \ldots, l_{\phi, s}$, and those after the previous $s$-th iteration step by $\nu_{s-1}, \ldots, l_{\phi, s-1}$. The basic geometry fields are then iterated via the equations

$$
\begin{aligned}
\nu_{s} & ={ }^{3} \Delta^{-1} S_{\nu, s-1}, \\
\alpha_{s} & ={ }^{2} \Delta^{-1} S_{\alpha, s-1}-\nu_{s}, \\
\beta_{s} & =\left({ }^{2} \Delta^{-1} S_{\beta, s-1}\right) /(r \sin \theta)-\nu_{s}, \\
M_{s}^{m} & =e^{-2\left(\alpha_{s}+\nu_{s}\right) 2} \Delta^{m}{ }_{n}{ }^{-1} S_{M, s-1}^{n}, \\
N_{s}^{a} & ={ }^{3} \Delta^{a}{ }_{b}{ }^{-1} S_{N, s-1}^{b},
\end{aligned}
$$

where $S_{\ldots, s-1}^{\ldots,}$ denotes the sources of equations (13-17) computed from the basic field values $\nu_{s-1}, \ldots, l_{\phi, s-1}$. The 
inverse Laplacians $\cdots \Delta_{\ldots-1}^{\cdots-1}$ appearing above are determined with Green functions according to the detailed description given further below (Sect. IIIB). Subsequently we evaluate the source of equation (33) using the newly computed values $\nu_{s}, \ldots, M_{s}^{\theta}$ of the basic geometry fields and the old values $p_{s-1}, \ldots, l_{\phi, s-1}$ of the basic matter fields, the outcome being called $S_{\chi_{0}, s-1}$. Next, we calculate

$$
\begin{aligned}
p_{s}= & H^{-1}\left[H\left(p_{\mathrm{c}}\right)+\left(\ln \left(u_{t, s-1}^{\mathrm{c}}\right)^{2}-\ln \left(u_{t, s-1}\right)^{2}\right) / 2\right. \\
& \left.\quad+I\left(\psi_{\mathrm{c}}\right)-I\left(\psi_{s-1}\right)\right] \\
\epsilon_{s}= & \epsilon\left(p_{s}\right), \\
\chi_{0}= & \left({ }^{3} \Delta^{-1}\left(S_{\chi_{0}, s-1} \cos \phi\right)\right) / \cos \phi \\
l_{\phi, s}= & L\left(\psi_{s-1}\right),
\end{aligned}
$$

where $u_{t, s-1}^{\mathrm{c}}$ is the value of the quantity $u_{t}^{\mathrm{c}}$ computed from the values of the basic fields $\nu_{s-1}, \ldots, l_{\phi, s-1}$.

\section{B. Green functions}

In the following, we address the inverse Laplacians ${ }^{2} \Delta^{-1},{ }^{3} \Delta^{-1},{ }^{2} \Delta^{m}{ }_{n}{ }^{-1}$ and ${ }^{3} \Delta^{a}{ }_{b}{ }^{-1}$ appearing in equations (39-44). Each of these inverse Laplacians comes from an equation having one of the forms

$$
\begin{aligned}
{ }^{2} \Delta \Phi & =S, \\
{ }^{3} \Delta \Phi & =S, \\
{ }^{2} \Delta^{m}{ }_{n} \Phi^{n} & =S^{n}, \\
{ }^{3} \Delta^{a}{ }_{b} \Phi^{b} & =S^{a},
\end{aligned}
$$

where $\Phi^{\cdots}$ and $S^{\cdots}$ are a potential and a source, respectively. To solve for the potential of equation (45), (46), ..., we have to compute the first, second, ... of the following integrals, shown in the same order as above [5]:

$$
\begin{aligned}
\Phi(\vec{x}) & =\int \mathrm{d}^{2} x^{\prime} \frac{S\left(\vec{x}^{\prime}\right)}{2 \pi / \ln \left|\vec{x}-\vec{x}^{\prime}\right|}, \\
\Phi(\vec{x}) & =-\int \mathrm{d}^{3} x^{\prime} \frac{S\left(\vec{x}^{\prime}\right)}{4 \pi\left|\vec{x}-\vec{x}^{\prime}\right|}+\ldots, \\
\Phi^{m}(r, \theta) & =\frac{\partial(r, \theta)^{m}}{\partial(x, z)^{n}} \int \mathrm{d}^{2} x^{\prime} \frac{\partial\left(x^{\prime}, z^{\prime}\right)^{n}}{\partial\left(r^{\prime}, \theta^{\prime}\right)^{o}} \frac{S^{o}\left(r^{\prime}, \theta^{\prime}\right)}{2 \pi / \ln \left|\vec{x}-\vec{x}^{\prime}\right|}, \\
\Phi^{a}(r, \theta) & =-\frac{\partial(r, \theta, \phi)^{a}}{\partial(x, y, z)^{b}} \int \mathrm{d}^{3} x^{\prime} \frac{\partial\left(x^{\prime}, y^{\prime} z^{\prime}\right)^{b}}{\partial\left(r^{\prime}, \theta^{\prime}, \phi^{\prime}\right)^{c}} \frac{S^{c}\left(r^{\prime}, \theta^{\prime}\right)}{4 \pi\left|\vec{x}-\vec{x}^{\prime}\right|},
\end{aligned}
$$

where

$$
\vec{x}=(x, z)=(r \sin \theta, r \cos \theta)
$$

for the 2-dimensional and

$$
\vec{x}=(x, y, z)=(r \sin \theta \cos \phi, r \sin \theta \sin \phi, r \cos \theta)
$$

for the 3-dimensional integrals (analogous for $\vec{x}^{\prime}$ ) together with the Jacobian determinants $\partial(\ldots)^{a} / \partial(\ldots)^{b}$. The dots appearing in equation (50) represent additional terms required for the boundary condition of the basic matter field $\chi_{0}$ and are given in Appendix F. In that Appendix, the main focus is on an expansion of the above integrals in terms of trigonometric functions and Legendre polynomials. Typically, only a few terms of the expansions are needed, and neglecting the remaining ones leads to a strong reduction of the computational cost necessary to evaluate the integrals.

\section{Slicing conditions}

According to Gourgoulhon et al. [6], the basic geometry fields $M^{m}$ and $N^{a}$ satisfy the following slicing conditions

$$
\begin{aligned}
\left(N^{2} M^{m}\right)_{|| m} & =0, \\
N_{\mid a}^{a} & =0,
\end{aligned}
$$

where the 2-and 3-covariant derivatives 'Il' and '"' are defined in Appendix B. A lengthy, but elementary calculation shows that these two slicing conditions can be rewritten as the vanishing flat-space 2 - and 3 -dimensional derivatives

$$
\begin{aligned}
\partial_{m} M_{e}^{m}+\frac{1}{r} M_{e}^{r} & =0, \\
\partial_{m} N_{e}^{m}+\frac{2}{r} N_{e}^{r}+\cot \theta N_{e}^{\theta} & =0,
\end{aligned}
$$

of the quantities

$$
\begin{aligned}
M_{e}^{m} & =e^{2(\alpha+\nu)} M^{m}, \\
N_{e}^{a} & =e^{2 \alpha+\beta} N^{a} .
\end{aligned}
$$

Contrary to an analytic one, a numeric evaluation of the Green functions of Sect. III B always produces values for the fields $M^{m}$ and $N^{a}$ which somewhat violate the slicing conditions. Therefore, we consider a 2- and 3dimensional Helmholtz decomposition and set the gradient parts of the two quantities $M_{e}^{m}$ and $N_{e}^{a}$ equal to zero such that the divergences of the remaining parts vanish as demanded by the slicing conditions. Such a procedure is valid, because when the fixed point iteration has converged, the gradient parts (which have to be set to zero) decrease with increasing numerical accuracy, i.e. with increasing grid resolution and number of trigonometric functions and Legendre polynomials used in the integral expansions of the Green functions.

\section{Final gauge}

Even now, the basic fields are not yet determined completely. There are still remaining gauge degrees of freedom left, because we can add an arbitrary constant to each of the potentials appearing in equations (45-48) without violating these equations:

$$
\Phi^{\cdots} \rightarrow \Phi^{\cdots}+\text { const } \cdots .
$$


For the 3-dimensional Poisson equations, the Green function

$$
{ }^{3} G\left(\vec{x}, \vec{x}^{\prime}\right)=-\frac{1}{4 \pi\left|\vec{x}-\vec{x}^{\prime}\right|}
$$

vanishes in the limit $\left|\vec{x}-\vec{x}^{\prime}\right| \rightarrow \infty$. Thus, we choose the potentials of these Poisson equations to vanish at infinity by setting the constant appearing in equation (52) equal to zero. However, for the 2-dimensional Poisson equations such a choice is not possible, as the Green function

$$
{ }^{2} G\left(\vec{x}, \vec{x}^{\prime}\right)=\frac{1}{2 \pi} \ln \left|\vec{x}-\vec{x}^{\prime}\right|
$$

is not bounded for $\left|\vec{x}-\vec{x}^{\prime}\right| \rightarrow \infty$. Therefore, we proceed as follows:

The potential $r \sin \theta(\beta+\nu)$ of the 2 -dimensional Poisson equation (41) obeys the Dirichlet boundary condition according to Appendix F. Hence, we set the constant in equation (52) equal to zero in this case, which completely fixes the field $\beta$. In case of the 2-dimensional Poisson equation (40), we choose the constant in equation (52) in such a manner that

$$
\alpha(r=0)=\beta(r=0) .
$$

For the remaining 2-dimensional Poisson equation (42), we have to consider only the Cartesian $z$-component $M_{e}^{z}$, because the $x$-component $M_{e}^{x}$ obeys the Dirichlet boundary condition, and we impose the condition

$$
M_{e}^{z}(r=0)=0
$$

\section{E. Circulation modes}

Eventually, we have a closer look at the function $f(\psi)$ introduced in equation (32). We follow the same approach as Eriguchi et al. [3] and limit ourselves to the power law

$$
f(\psi)=k \psi^{n}
$$

with some constant $k$ and the exponent

$$
n=0,1 \text {. }
$$

For the case

$$
f(\psi)=k \psi
$$

we find different meridional circulation modes $\psi_{m}$ (with $m=0,1,2, \ldots)$, as in the Newtonian case [3]. The distributions of the field $\chi_{0}$ belonging to these modes look similar to the ones displayed in Fig. 1 but are somewhat deformed.

Unfortunately, when nearly having reached one of the higher circulation modes $\psi_{1}, \psi_{2}, \ldots$ during the fixed point iteration, the fixed point iteration method always starts to converge to the fundamental mode $\psi_{0}$. In order to obtain the higher modes, we therefore projected the lower ones away. For that purpose, we assume that we have already evaluated the first $m_{\mathrm{e}}-1$ modes, i.e. we know $\psi_{m}$ for $m=0,1, \ldots, m_{\mathrm{e}}-1$. Then, the fixed point iteration leads to the $m_{\mathrm{e}}$-th mode by replacing

$$
\psi \rightarrow \psi-\sum_{m=1}^{m_{\mathrm{e}}-1} C_{m} \psi_{m}
$$

at every iteration step, with adequately chosen coefficients $C_{m}$. If an orthogonality relation

$$
\int_{0}^{\infty} \mathrm{d} r \int_{0}^{\pi} \mathrm{d} \theta \sqrt{h} W_{m}(r, \theta) \psi_{m} \psi_{m^{\prime}}=\delta_{m m^{\prime}}
$$

with the weight function $W_{m}(r, \theta)$ exists, the coefficients $C_{m}$ are given by

$$
C_{m}=\frac{\int_{0}^{\infty} \mathrm{d} r \int_{0}^{\pi} \mathrm{d} \theta \sqrt{h} W_{m}(r, \theta) \psi \psi_{m}}{\int_{0}^{\infty} \mathrm{d} r \int_{0}^{\pi} \mathrm{d} \theta \sqrt{h} W_{m}(r, \theta) \psi_{m} \psi_{m}}
$$

where $h=\operatorname{det} h_{a b}$. However, we neither know whether an orthogonality relation exists nor do we know the weight functions $W_{m}(r, \theta)$. After some experimenting, we found that the choice

$$
W_{m}(r, \theta)=\epsilon
$$

is sufficient to achieve a convergence to higher modes. This does not necessarily mean that (56) is the correct weight function, but it could be very close to it.

In addition to the usage of (56), we perform the following steps in the GRNS code: The pressure distribution of the solutions investigated in this work is always equatorially symmetric. However, in our treatment, equatorial symmetry is not guaranteed exactly due to the limited numerical accuracy. Therefore, equatorial asymmetry may increase during the fixed point iteration, eventually leading to an undesired meridional circulation mode. In order to prevent this, we symmetrize the pressure distribution at every iteration step. A similar method is performed for the basic field $\chi_{0}$, which has either even or odd parity depending on the considered mode.

\section{F. GRNS}

We have implemented GRNS under Linux in $\mathrm{C}++$, and it possesses an OpenGL visualization interface, which allows the user to supervise the fixed point iteration. The user has full control over the iteration, which can be stopped and restarted at any time. The user can select any of the physical fields either when the iteration is stopped or even when it is running, and display it on the screen. It is also possible to visualize the flow of the neutron star fluid in real time and to see how the neutron star surface changes during the iteration. These code features are of advantage when analyzing the stability of 
GRNS, and they were also very helpful in debugging the code.

We used a numerical grid of 150 radial and 156 angular zones to compute the models discussed in Sect. IV. For the sums arising from the expansion of the integrals (see Appendix F), we chose the upper limit

$$
\sum_{l=\ldots}^{\infty} \rightarrow \sum_{l=\ldots}^{l_{\max }}
$$

where $l_{\max }=10$ for all models of Sect. IV. This limits the computational cost, and we are able to compute individual circulation modes within about a minute on a current single core CPU. In Appendix G, we discuss the convergence behavior and consistency tests of GRNS.

\section{RESULTS}

\section{A. Reference model}

The results presented in the following were obtained with the usual polytropic equation of state

$$
\begin{aligned}
p & =K \rho^{\Gamma}, \\
\epsilon & =\rho+\frac{p}{\Gamma-1},
\end{aligned}
$$

where $K$ is the polytropic constant, $\rho$ the rest mass density, and $\Gamma$ the polytropic exponent, respectively. From this equation of state its is readily seen that

$$
\epsilon=\left(\frac{p}{K}\right)^{\frac{1}{\Gamma}}+\frac{p}{\Gamma-1}
$$

We keep the maximum absolute value of the stream function $\psi$ fixed at the value $\psi_{\max }$ such that it does not matter which value we choose for the constant $k$ in equation (53). The fundamental mode of each neutron star model is then unambiguously specified by the following parameters

$$
n, p_{\mathrm{c}}, \rho_{\mathrm{c}}, \Gamma, \psi_{\max }, L(\psi)
$$

i.e. by the exponent appearing in equation (53), the central pressure and the central density (which fixes the polytropic constant $K$ ) of the initial guess, the polytropic exponent, the maximum absolute value of the stream function, and by the distribution (28) of the specific angular momentum. As a reference model, we choose a non-rotating neutron star with parameters

$$
\begin{aligned}
n & =1, \\
p_{\mathrm{c}} & =9.1 \cdot 10^{34} \mathrm{erg} / \mathrm{cm}^{3}, \\
\rho_{c} & =7.9 \cdot 10^{14} \mathrm{~g} / \mathrm{cm}^{3}, \\
\Gamma & =2, \\
\psi_{\max } & =3 \cdot 10^{34} \mathrm{~g} / \mathrm{s}, \\
L(\psi) & =0,
\end{aligned}
$$

circulating at its fundamental mode. This set of parameters corresponds to a neutron star with the following properties:

$$
\begin{aligned}
\bar{R} & =15.6 \mathrm{~km}, \\
\mathcal{M} & =1.51 M_{\odot}, \\
\bar{v}_{\text {circ }} & =1043 \mathrm{~km} / \mathrm{s} .
\end{aligned}
$$

The average radius $\bar{R}$ is the average proper radius

$$
R_{C}=\int_{C} \mathrm{~d} r e^{\alpha}
$$

averaged over all angles $\theta$ (over all radial paths $C$ ). We compute the rest mass via [11]

$$
\mathcal{M}=\int_{V} \mathrm{~d} r \mathrm{~d} \theta \mathrm{d} \phi \sqrt{h} N u^{t} \rho,
$$

where $V$ is the volume of the star and the average circulation velocity is given by

$$
\bar{v}_{\mathrm{circ}}=\left\langle\sqrt{\left(v^{r}\right)^{2}+\left(r v^{\theta}\right)^{2}}\right\rangle
$$

The basic fields of the reference model are shown in Fig. 2. The 3-lapse $\log$ arithm $\nu$ is one of the two contributions (the other one comes from the 3-shift $N^{a}$ ) to the time dilation, which is strongest at the center of the neutron star. The 2-conformal factor logarithm $\alpha$ represents the stretching of space in meridional planes and is also strongest in the center. We do not display the basic geometry field $\beta$ in Fig. 2, because its values are not significantly different from those of the field $\alpha$. The 3 -shift $N^{a}$ represents the dragging of spacetime caused by the flow of the neutron star fluid. Since the reference model is circulating, this leads to the vortex visible in the lower left panel of Fig. 2. The total energy density $\epsilon$ is also not shown in that figure, because it is correlated to the pressure $p$ by the trivial analytic relation (58). Note that in the lower right panel of Fig. 2 the outermost contour is the surface of the neutron star. As the values on the axes of Fig. 2 do not refer to proper but to coordinate distances, the average neutron star radius suggested by the contour is somewhat smaller than the actually correct value (59), which takes the curvature of spacetime into account. The 2-shift $M^{m}$ and specific angular momentum $l_{\phi}$ vanish for the reference model, because it does not rotate. Instead of considering the modified stream function $\chi_{0}=\psi /(r \sin \theta)$, we show the stream function $\psi$ itself in the top left panel of Fig. 3, as the contours of the stream function $\psi$ are stream lines of the meridional flow. Note that the stream function of the reference model contains only a single vortex.

The distributions of the 3-lapse $\nu$, the 2-conformal factor $\alpha$ and the pressure $p$ given in Fig. 2 do not show any significant difference when compared to the TolmanOppenheimer-Volkoff solution used to construct the initial guess for the fixed point iteration. The only relevant 

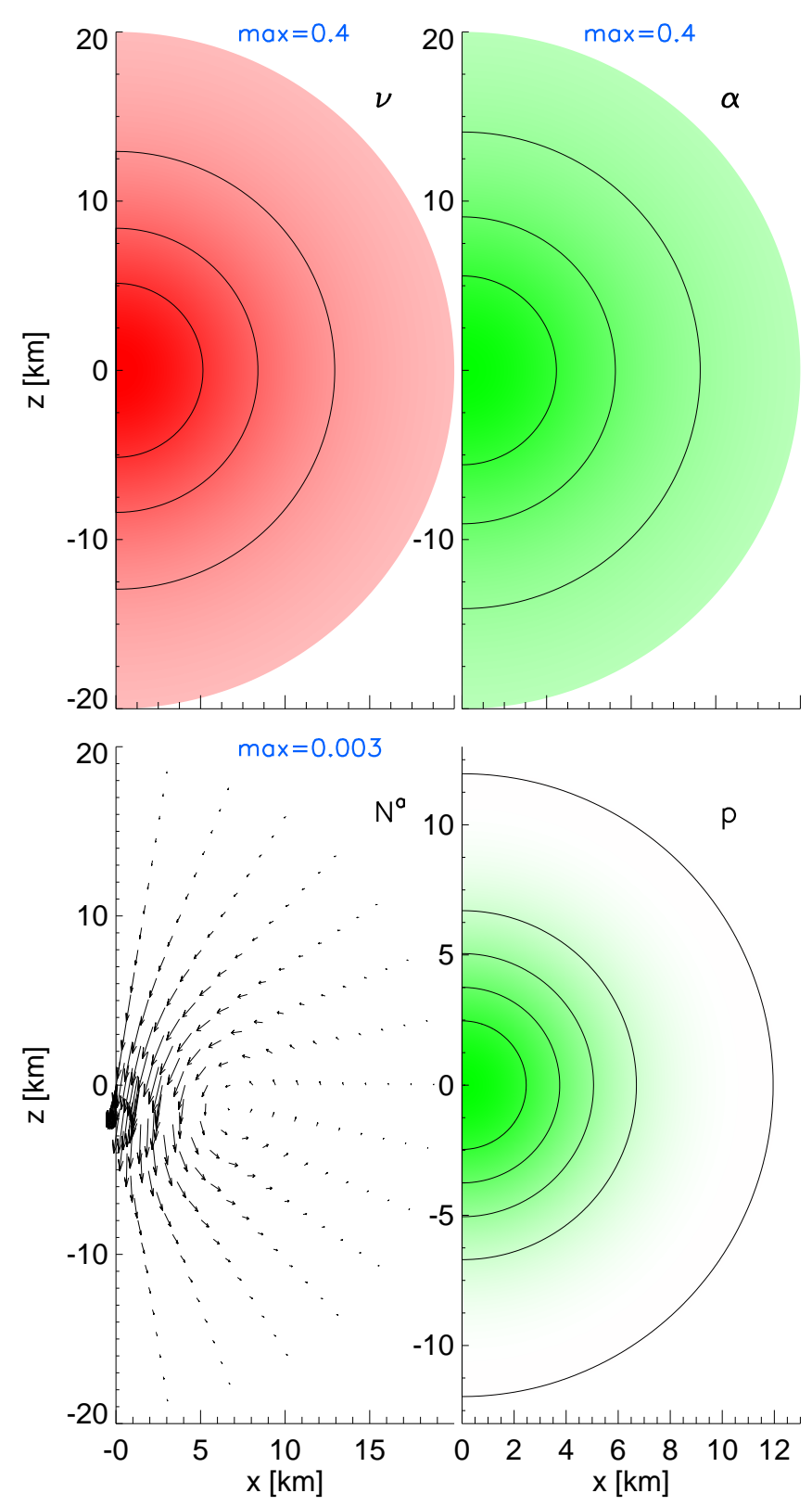

FIG. 2: Basic fields of reference model. The four panels show the 3-lapse logarithm $\nu$, the 2-conformal factor logarithm $\alpha$, the 3 -shift $N^{a}$ and the pressure for our reference model. The color coding of the three scalar plots is the same one as in Fig. 1, where the maximum absolute field value $\max$ is displayed at the top of each panel. For the vector plot, the quantity $\max$ denotes the maximum vector length (note that all vectors lie within the paper plane).

changes are that the 3 -shift $N^{a}$ and the stream function $\psi$, which vanish for the Tolman-Oppenheimer-Volkoff solution, display the distributions shown in the lower, left panel of Fig. 2 and the upper left panel of Fig. 3, respectively.

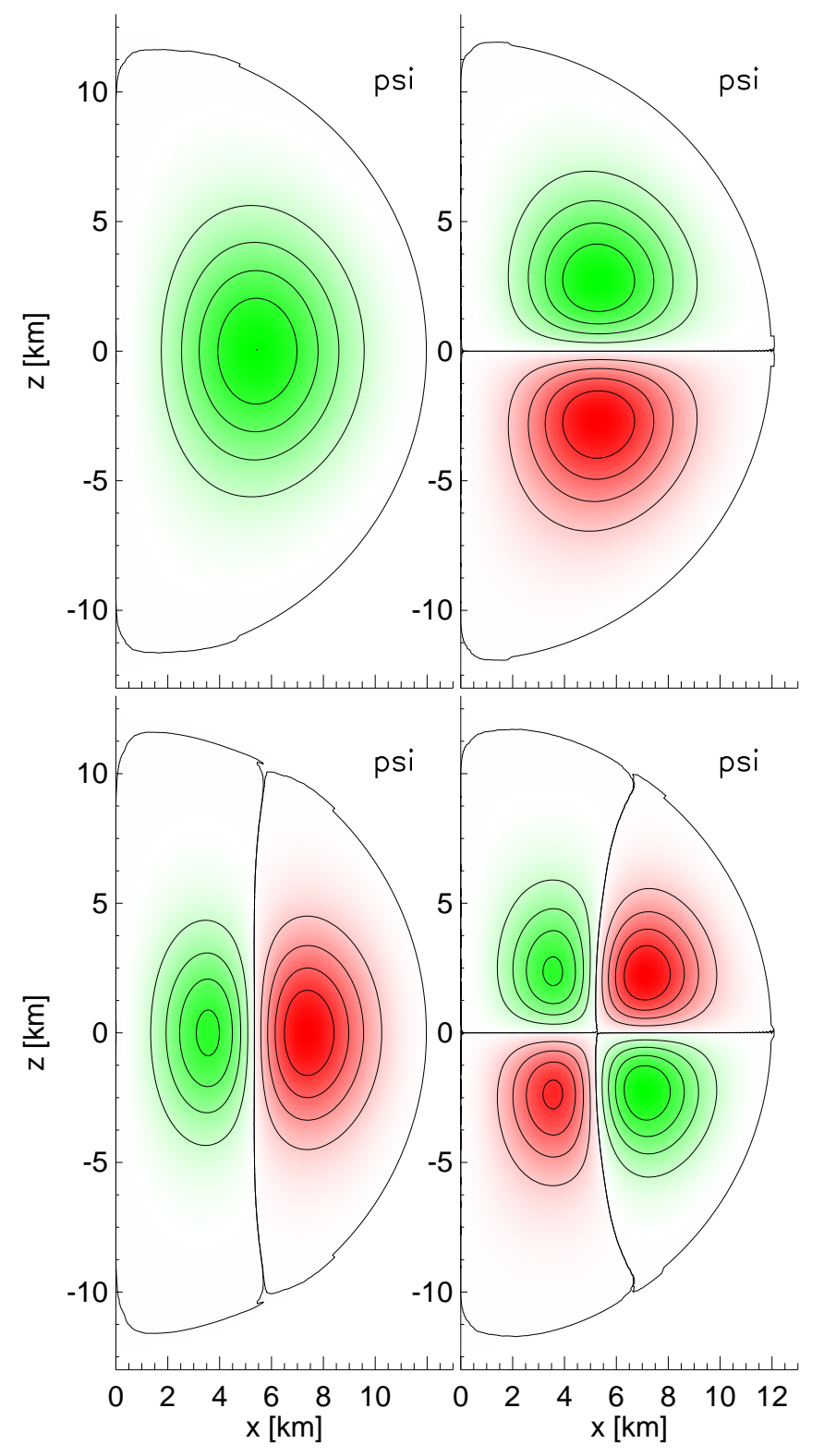

FIG. 3: Circulation modes - Part 1. The panels show the stream lines of the first four meridional circulation modes of the reference model. The color coding is the same as in Fig. 1. Note that red and green refer to clockwise and counterclockwise motion, respectively. The kinks visible at the neutron star surface are a result of the finite numerical resolution and the use of contour plots.

\section{B. Higher circulation modes}

The other three panels in Fig. 3 show higher circulation modes with additional vortices. Actually, each vortex in the stream function $\psi$ is accompanied by a vortex in the 3-shift $N^{a}$ (not shown for the higher modes). Let us now compare the four modes shown in Fig. 3 with the four initial guesses of Fig. 1. With this in mind, it 
does not matter that both figures show different fields, because the difference between the stream function $\psi$ and the modified stream function $\chi_{0}$ is merely a factor $r \sin \theta$. Such a factor causes deformations of the vortices, but their number and orientations remain unaffected. Then, it seems as if the top left initial guess of Fig. 1 leads to the top left mode of Fig. 3, and so on. However, there is no such one-to-one correlation. Instead, it can, for instance, occur that the four modes of Fig. 3 are produced by the four initial guesses $\mathcal{M}_{r}=1$ and $\mathcal{M}_{\theta}=1,2,3,4$. Actually, it does not matter too much how the initial guesses are chosen. It is only important that they are somehow different, and to improve the fixed point iteration, we have made a choice that is at least similar to the expected outcomes.

In total, we are able to compute 16 modes for the reference model (including the fundamental mode) before GRNS fails due to too large numerical errors, i.e. we find a much larger set of modes than in Eriguchi et al. [3]. Fig. 4 shows some additional higher modes. The lower right panel of that figure represents the highest mode obtained where numerical errors do not yet have a significant impact on the shape of the vortices. Looking at Figs. 3 and 4 , it is obvious that the circulation modes constitute a 2-dimensional mode set.

\section{Surface deformations}

A closer look at the surface of the neutron star in Fig. 5 reveals that surface deformations of about half a percent of the average neutron star radius are present. The shape of these deformations depends on the vortices in the vicinity of the surface. Vortices deeper inside of the neutron star have only a small impact on the deformations. In Eriguchi et al. [3], the numerical resolution was too small to resolve such properties. However, it was determined that for the fundamental mode with only a single vortex the meridional circulation causes the neutron star to become slightly prolate.

The strength of the deformations depends also on the velocity of the fluid in the vortices, and thus on the maximum absolute value $\psi_{\max }$ of the stream function. To test the limits, we increased the value of $\psi_{\max }$ in GRNS as far as possible. The choice $\psi_{\max }=3 \cdot 10^{34} \mathrm{~g} / \mathrm{s}$ for the reference model is already close to what is possible with GRNS, and we were only able to increase that value to about $\psi_{\max }=10^{35} \mathrm{~g} / \mathrm{s}$, above which numerical errors grow dramatically. However, even at the limiting value, the surface deformations do not exceed about one percent of the average neutron star radius.

\section{Circulation velocities}

For the choice $\psi_{\max }=10^{35} \mathrm{~g} / \mathrm{s}$, the average circulation velocity of the reference model is $\bar{v}_{\text {circ }}=3431 \mathrm{~km} / \mathrm{s}$, whereas for a very slowly circulating model with $\psi_{\max }=$

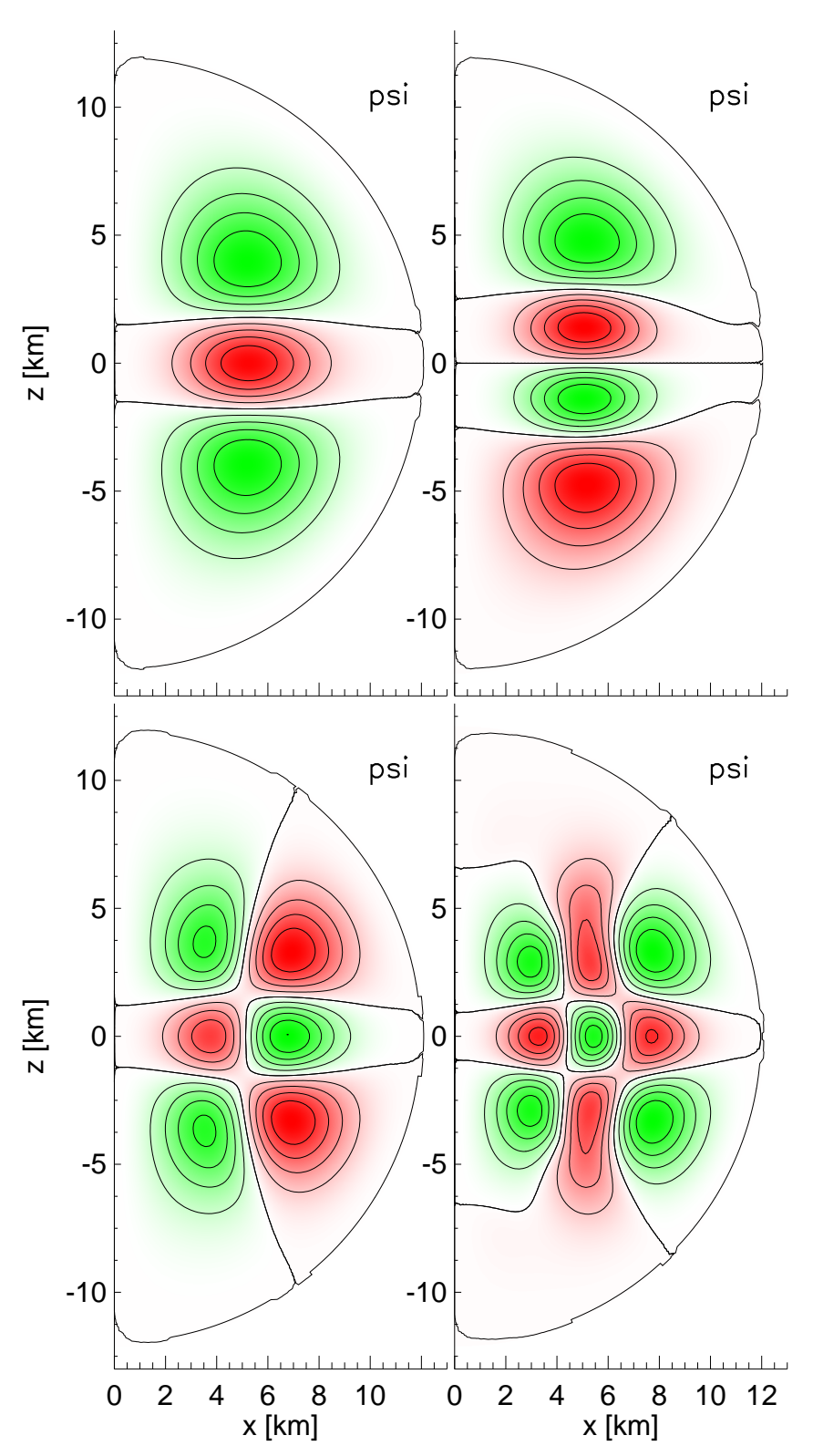

FIG. 4: Circulation modes - Part 2

$3 \cdot 10^{34} \mathrm{~g} / \mathrm{s}$ the average circulation velocity is only $\bar{v}_{\text {circ }}=$ $10 \mathrm{~km} / \mathrm{s}$. Actually, it is a good approximation to say that the average circulation velocity $\bar{v}_{\mathrm{c}}$ scales linearly with the maximum absolute value $\psi_{\max }$ of the stream function. However, for the average radius and the mass of the neutron star, we do not find any significant changes when the circulation velocity changes, within the range considered here.

Tab. I lists the average velocities $\bar{v}_{\text {circ }}$ of the circulation modes. For that purpose, we use a mode numbering similar to Fig. 1: the first mode number in Tab. I gives the number of vortices in $x$-direction, and the second one in $z$-direction. The velocity $\bar{v}_{\text {circ }}$ obviously increases with the mode numbers, which is a consequence of the orthog- 


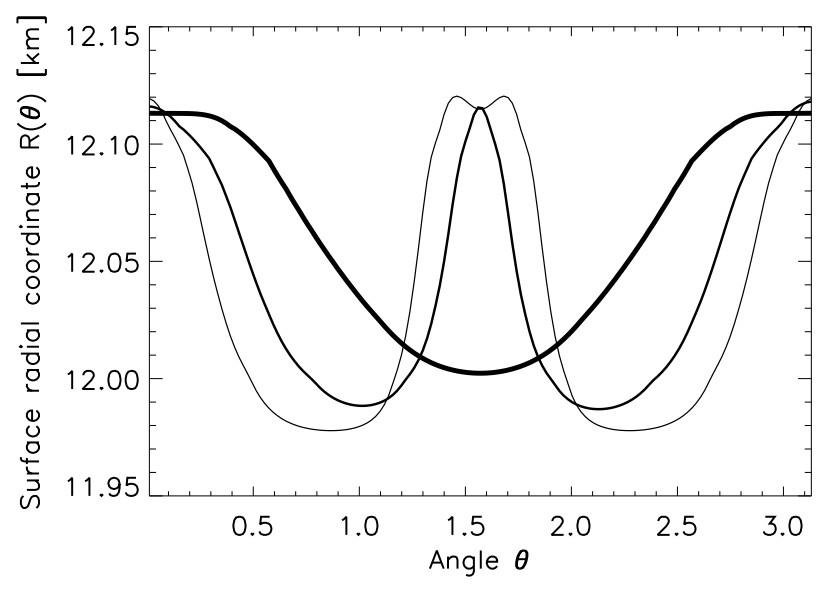

FIG. 5: Surface deformations. The figure shows the radial coordinate $R(\theta)$ of the neutron star surface depending on the angle $\theta$. From thick to thin, the three lines refer to the upper left and upper right mode of Fig. 3, and the upper left mode of Fig. 4.

TABLE I: Average circulation velocities. The table shows the average velocities $\bar{v}_{\text {circ }}$ for the eight circulation modes of Figs. 3 and 4.

\begin{tabular}{cccccccccc}
\hline \hline Mode & $(1,1)$ & $(1,2)$ & $(1,3)$ & $(1,4)$ & $(2,1)$ & $(2,2)$ & $(2,3)$ & $(3,3)$ \\
\hline $\bar{v}_{\text {circ }}[\mathrm{km} / \mathrm{s}]$ & 1032 & 1296 & 1569 & 1778 & 1364 & 1661 & 2002 & 2444 \\
\hline
\end{tabular}

onality relation (55). As the average circulation velocity $\bar{v}_{\text {circ }}$ rises with the maximum absolute value $\psi_{\max }$ of the stream function, changing the orthogonality relation (55) by inserting an appropriate factor $D_{m}$ in the integrand, it is possible to create new modes with the same velocity $\bar{v}_{\text {circ }}$, but a different maximum $\psi_{\max }$. Because it is not easy to determine the factor $D_{m}$, our method is more practical.

\section{E. Constant $f(\psi)$}

Eriguchi et al. [3] investigated both cases (54). Therefore, we now take the reference model and set the parameter $n=0$ such that we obtain

$$
f(\psi)=k .
$$

For this case, we do not find a collection of circulation modes but only a single solution, which is shown in Fig. 6. There, we see that compared to the upper left panel of Fig. 3 the stream function is slightly deformed. In addition to that, we find that the average circulation velocity becomes

$$
\bar{v}_{\text {circ }}=1366 \mathrm{~km} / \mathrm{s} \text {, }
$$

while the average proper radius $\bar{R}$ and the mass $\mathcal{M}$ of the neutron star are the same as for the reference model.

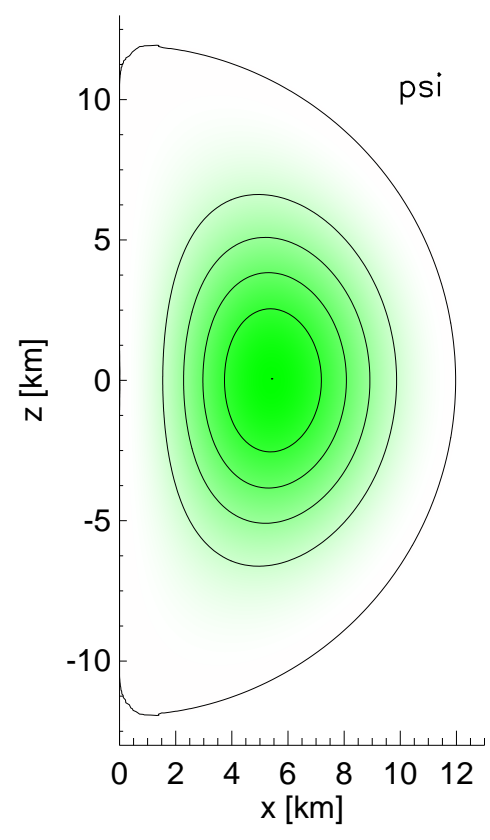

FIG. 6: Constant $f(\psi)$. Stream function $\psi$ for the case $n=0$. The color coding is the same one as in Fig. 1 .

\section{F. Rotating neutron star}

In contrast to Eriguchi et al. [3], we have also computed rotating neutron star models with meridional circulation. For that purpose, we set the specific angular momentum $L(\psi)$ to a non-vanishing value. The simplest choice would be

$$
L(\psi)=\text { const } \neq 0,
$$

where the neutron star rotates rapidly near the rotation axis. Eriguchi et al. [3] exclude such models, because the rotation velocity becomes infinite when approaching the rotation axis in Newtonian configurations. In general relativity, the situation is more complicated, because spacetime itself can be dragged by the neutron star fluid. We are not able to present stringent analytical reasons which exclude condition (60), but simulations obeying that condition do not seem to converge for higher numerical resolutions and have to be considered as invalid neutron star models.

Therefore, we consider the next simplest case

$$
L(\psi)=\text { const } \cdot \psi \text {. }
$$

For the choice

$$
L(\psi)=3 \cdot 10^{14} \frac{\psi}{\psi_{\max }} \frac{\mathrm{cm}^{2}}{\mathrm{~s}},
$$

we find the average circulation and rotation velocities

$$
\begin{aligned}
\bar{v}_{\text {circ }} & =1168 \mathrm{~km} / \mathrm{s}, \\
\bar{v}_{\text {rot }} & =431 \mathrm{~km} / \mathrm{s},
\end{aligned}
$$


where the latter is the average value of

$$
v_{\text {rot }}=r \sin \theta v^{\phi} \text {. }
$$

When compared to the reference model, the average radius and the mass do not show significant changes, just as most basic fields. Two exceptions are the specific angular momentum $l_{\phi}$ and the 2 -shift $M^{m}$, which vanish for the reference model, whereas for the rotating model (61) their distributions look like as displayed in Fig. 7. They now exhibit a vortex, similar to the stream function $\psi$ and the 3-shift $N^{a}$. In contrast to the reference model, the 3-shift $N^{a}$ vector does not only lie within the meridional plane as shown in the lower left panel of Fig. 2, but it has also contributions perpendicular to that plane for the rotating model (not displayed in this paper).

Having investigated model (61), where the circulation and rotation velocities are of the same order of magnitude, the question arises what happens when the rotational velocity is much smaller or much larger than the circulation velocity. Since the reference model itself is non-rotating, we continuously increased its rotation velocity from zero to the value (62) by changing the constant in relation (61). Currently, GRNS fails to compute models where the average rotation velocity is larger than about the value (62). Actually, one would expect that there are at least a few models where the rotation velocity is much larger than the circulation velocity, and possible improvements in the numerical method could allow their computation.

\section{G. Higher modes with circulation and rotation}

There are not only higher circulation modes for nonrotating configurations, like the reference model, but also for rotating models. In this case, the difference between the fundamental mode and the higher modes is similar to what has been discussed in Sect. IV B. All of the basic fields containing a single vortex for the fundamental mode, i.e. the four fields $\psi, N^{a}, l_{\phi}, M^{m}$, exhibit the same number of vortices for a certain higher mode. These vortices are always roughly located at the same spatial position for the four fields. Similar to the non-rotating configurations, the modes where both circulation and rotation are present turn out to be a 2-dimensional mode collection.

Fig. 8 shows radial profiles of the specific angular momentum (per unit rest mass)

$$
j=\frac{\epsilon+p}{\rho} u_{\phi}
$$

(different from the specific angular momentum $l_{\phi}$, which is defined per unit energy) for the fundamental and one higher mode belonging to the rotating model (61). There, we do not only recognize combined co- and counterrotation, but also that in parts of the star $\mathrm{dj} / \mathrm{dr}<0$, which implies that the flow does not satisfy Solberg's criterion for local stability (see [12] for a proof in GR).

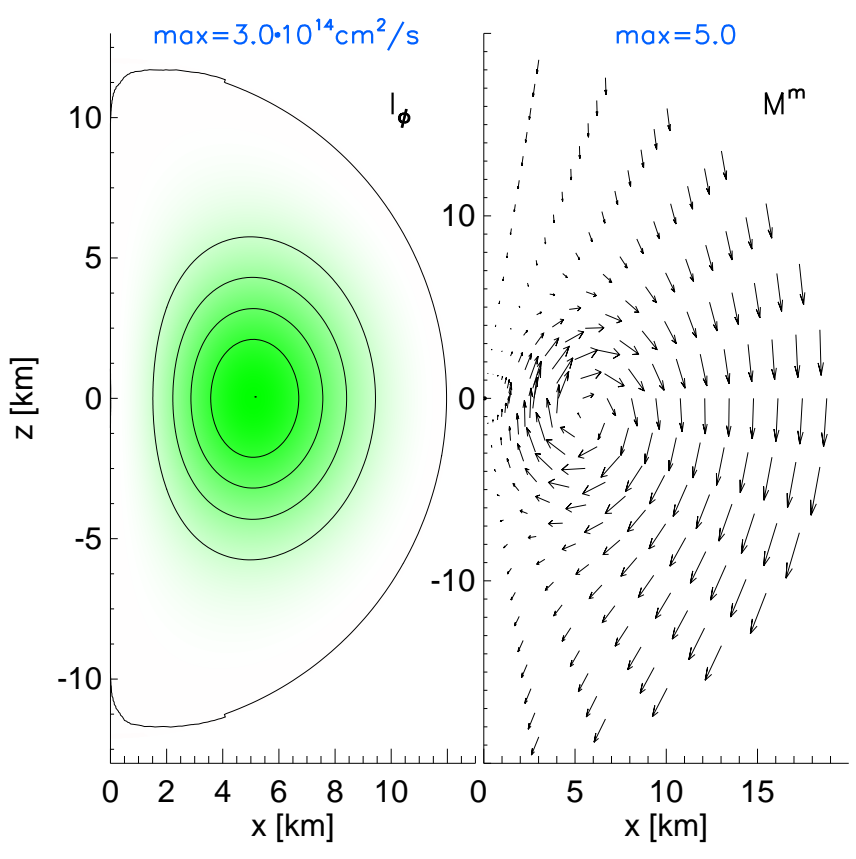

FIG. 7: Rotating neutron star. Specific angular momentum $l_{\phi}$ and the 2 -shift $M^{m}$ for the rotating model (61). The color coding of the contour plot is the same one as in Fig. 1. For the vector plot, the quantity max denotes the maximum vector length.

\section{CONCLUSIONS}

We computed the first stationary, axisymmetric neutron star models with meridional circulation in the framework of general relativity. For that purpose, we have developed GRNS, a new code that uses a fixed point iteration method starting from a Tolman-OppenheimerVolkoff like initial configuration, similar to RNS, written by N. Stergioulas.

We found meridional circulation modes like Eriguchi et al. [3] in the Newtonian framework. However, by using a fixed point iteration instead of a Newton-Raphson one, we were able to automatize the process of computing such modes. As a result, we identified a much larger set of modes than these authors. Our study shows that the circulation models form a two-dimensional set.

The circulation modes differ by a varying number of vortices in the stream lines of the fluid. These vortices cause deformations of the surface of the neutron star. The deformations are influenced strongest by the vortices in the vicinity of the surface, and their influence rises with the value of the circulation velocity. We found surface deformations of the order of one percent for expected maximal circulation velocities of about $1000 \mathrm{~km} / \mathrm{s}$. However, the radius and mass of the neutron star do not significantly depend on the circulation velocities.

In contrast to Eriguchi et al. [3], we also computed rotating neutron star models with meridional circulation. 


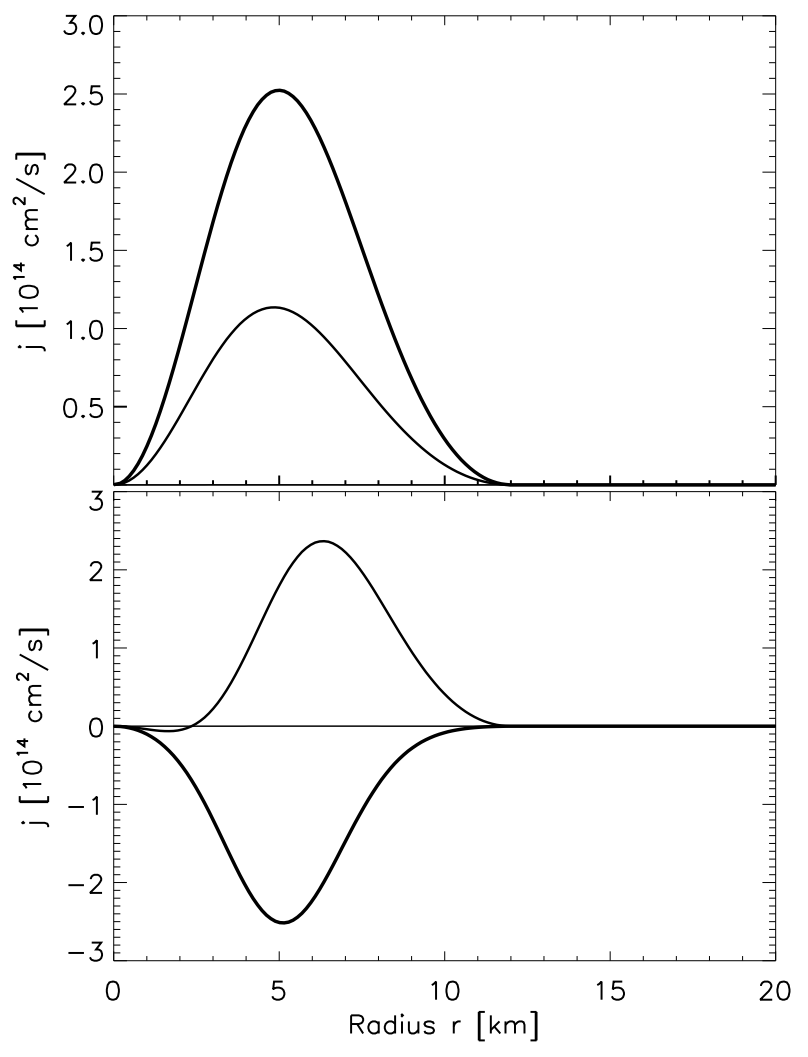

FIG. 8: Specific angular momentum. The two panels show radial profiles of the specific angular momentum $j$ for two different modes of the rotating model (61) at angles $\theta=0$ (thinnest curve), $\theta=\frac{\pi}{4}$, and $\theta=\frac{\pi}{2}$ (thickest curve), respectively. The upper panel belongs to the fundamental mode, displayed in Fig. 7, and the lower one to one of the higher modes. The specific angular momentum $l_{\phi}$ and the stream function $\psi$ of this higher mode exhibit a vortex distribution as in the upper, left panel of Fig. 4.

For such models, the rotation velocity is highest in the center of the vortices, and vanishes at the surface of the neutron star. In addition to that, we were unable to find rotating neutron star models, where the rotation velocity is significantly larger than the circulation velocity. We are not sure whether this is possibly caused by our symmetry assumptions.

There are clear perspectives for a future application of the outcomes of this investigation. Perturbing the obtained modes, a dynamical evolution of the neutron star could show the influence of meridional circulations on gravitational waves, for which a direct experimental detection is expected in the near future. Another application is investigating the influence of meridional circulations on neutron star oscillations. Both methods offer a way to determine by means of observations whether meridional circulations are present in (young) neutron stars. At the current stage we are unable to decide how common such circulations are in nature, because with our study we did not evaluate stability criteria for the circulation modes.

In the near future, the two most important extensions of this investigation will be changing the topology to a toroidal one and to include magnetic fields. We have already presented some first thoughts in that direction in the Appendix finding that the field equation for the specific angular momentum is strongly affected by the presence of a magnetic field.

\section{ACKNOWLEDGMENTS}

We are grateful to Eric Gourgoulhon for useful comments. This work was supported by the DAAD via an IKYDA German-Greek research travel grant, by the Collaborative Research Center on Gravitational Wave Astronomy of the Deutsche Forschungsgemeinschaft (DFG SFB/Transregio 7), and by CompStar, a Research Networking Programme of the European Science Foundation.

\section{Appendix A: Corrected geometry equations}

In our investigation, we use equations (B3-B7) of Gourgoulhon et al. [6], which contain minor errors in the three equations (B3), (B4a), and (B4b). We found these errors by thoroughly verifying all the mathematical equations in that paper. For equation (B3), this was done by hand, and similar to Gourgoulhon et al. [6] we used the computer algebra program Mathematica to validate the rather lengthy equations (B4a) and (B4b). In the following, we will give the three corrected equations. Due to their length, we will not list them completely here, but show only where the corrections appear. Several new mathematical quantities are introduced during the derivation of the equations, which will not be defined here. Instead, we refer to Gourgoulhon et al. [6], whose notation and conventions are adopted by us, except for the differently chosen indices (1).

Equation (B3) of Gourgoulhon et al. [6] has the form

$$
\ldots=4 \pi\left(E+S_{a}^{a}\right)+K_{a b} K^{a b}+\frac{L^{2}}{2},
$$

where the term $2 m^{r} m^{\theta} \nu_{, r} \nu_{, \theta}$ is missing on the left hand side, i.e. the correct equation (B3) reads

$$
\ldots+2 m^{r} m^{\theta} \nu_{, r} \nu_{, \theta}=4 \pi\left(E+S_{a}^{a}\right)+K_{a b} K^{a b}+\frac{L^{2}}{2} .
$$

In the last line of the left hand side of equation (B4a) of Gourgoulhon et al. [6], there appears the expression

$$
\ldots+\frac{m^{\theta}}{M}\left[M_{, \theta}^{r}\left(\mu, \theta-4 \alpha_{, \theta}\right)-\frac{M_{, \theta \theta}^{r}}{M}\right] \ldots
$$

Here, the presence of the 2-lapse $M$ in the squared bracket is an error, i.e. the correct equation (B4a) has 
the form

$$
\ldots+\frac{m^{\theta}}{M}\left[M_{, \theta}^{r}\left(\mu_{, \theta}-4 \alpha_{, \theta}\right)-M_{, \theta \theta}^{r}\right] \ldots .
$$

The second but last line of the left hand side of equation (B4b) of Gourgoulhon et al. [6] reads

$$
\ldots \frac{m^{\theta}}{M}\left[\ldots-\frac{M_{, r \theta}^{r}}{M}-2 \frac{M_{, \theta \theta}^{\theta}}{M}-2 \frac{A_{, \theta \theta}}{A}\right] \ldots .
$$

The presence of the 2-lapse $M$ in the squared bracket is an error, and the third term $-2 A_{, \theta \theta} / A$ has to be deleted such that the correct equation (B4b) reads

$$
\ldots \frac{m^{\theta}}{M}\left[\ldots-M_{, r \theta}^{r}-2 M_{, \theta \theta}^{\theta}\right] \ldots .
$$

\section{Appendix B: Flat-space Laplacians}

In the following, we consider 2- and 3-dimensional Laplacians. For that purpose, we use the inverse 2- and 3 -metrices $k^{m n}$ and $h^{a b}[6]$, and the Christoffel symbols of the second

$$
\begin{aligned}
{ }^{2} \Gamma_{n o}^{m} & =k^{m p} \Gamma_{p n o}, \\
{ }^{3} \Gamma_{b c}^{a} & =h^{a d} \Gamma_{d b c}
\end{aligned}
$$

and of the first kind

$$
\Gamma_{\alpha \beta \gamma}=\frac{1}{2}\left(\partial_{\beta} g_{\alpha \gamma}+\partial_{\gamma} g_{\beta \alpha}-\partial_{\alpha} g_{\beta \gamma}\right) .
$$

These Christoffel symbols allow us to compute the 2- and 3 -covariant derivatives [6] of a tensor $T_{b \ldots}^{a \ldots}$ :

$$
\begin{aligned}
T_{n \ldots \mid j p}^{m \ldots} & =\partial_{p} T_{n \ldots}^{m \ldots}+{ }^{2} \Gamma_{q p}^{n} T_{m \ldots}^{q \ldots \ldots}+\ldots-{ }^{2} \Gamma_{n p}^{q} T_{q \ldots}^{m \ldots}-\ldots, \\
T_{b \ldots \mid c}^{a \ldots} & =\partial_{c} T_{b \ldots}^{a \ldots}+{ }^{3} \Gamma_{d c}^{a} T_{b \ldots}^{d \ldots}+\ldots-{ }^{3} \Gamma_{b c}^{d} T_{d \ldots}^{a \ldots}-\ldots
\end{aligned}
$$

Then, limiting ourselves to a scalar $\Phi$ and a vector $\Phi^{a}$, we construct the following Laplacians:

$$
\begin{aligned}
k^{n p} \Phi_{|| n p} & ={ }^{2} \Delta \Phi, \\
h^{b c} \Phi_{\mid b c} & ={ }^{3} \Delta \Phi, \\
k^{n p} \Phi^{m}{ }_{\mid n p} & ={ }^{2} \Delta^{m}{ }_{n} \Phi^{n}, \\
h^{b c} \Phi_{\mid b c}^{a} & ={ }^{3} \Delta^{a}{ }_{b} \Phi^{b} .
\end{aligned}
$$

For flat-space, we choose

$$
\begin{aligned}
k_{m n} & =\operatorname{diag}\left(1, r^{2}\right), \\
h_{a b} & =\operatorname{diag}\left(1, r^{2}, r^{2} \sin ^{2} \theta\right),
\end{aligned}
$$

such that a straightforward calculation gives the two well known scalar Laplacians

$$
\begin{aligned}
& { }^{2} \Delta=\partial_{r}^{2}+\frac{1}{r^{2}} \partial_{\theta}^{2}+\frac{1}{r} \partial_{r}, \\
& { }^{3} \Delta=\partial_{r}^{2}+\frac{1}{r^{2}} \partial_{\theta}^{2}+\frac{2}{r} \partial_{r}+\frac{\cot \theta}{r^{2}} \partial_{\theta}+\frac{1}{r^{2} \sin ^{2} \theta} \partial_{\phi}^{2},
\end{aligned}
$$

and the two less familiar vector Laplacians

$$
\begin{aligned}
{ }^{2} \Delta_{n}^{m} & =\delta_{n}^{m 2} \Delta+\left(\begin{array}{cc}
-\frac{1}{r^{2}} & -\frac{2}{r} \partial_{\theta} \\
\frac{2}{r^{3}} \partial_{\theta} & \frac{2}{r} \partial_{r}
\end{array}\right), \\
{ }^{3} \Delta_{b}^{a} & =\delta_{b}^{a 3} \Delta+\left(\begin{array}{ccc}
-\frac{2}{r^{2}} & -\frac{2}{r} \partial_{\theta}-2 \frac{\cot \theta}{r} & 0 \\
\frac{2}{r^{3}} \partial_{\theta} & \frac{2}{r} \partial_{r}+\frac{1-\cot ^{2} \theta}{r^{2}} & 0 \\
0 & 0 & m_{33}
\end{array}\right),
\end{aligned}
$$

with the matrix element $m_{33}=\frac{2}{r} \partial_{r}+2 \frac{\cot \theta}{r^{2}} \partial_{\theta}$. Note that equation (B1) holds only in case of axisymmetry, where the azimuthal derivatives $\partial_{\phi}$ of the Laplacians ${ }^{3} \Delta^{a}{ }_{b}$ and ${ }^{3} \Delta$ vanish.

\section{Appendix C: Energy equation}

In this appendix, we show that for our symmetry assumptions it is possible to write the energy equation (19) as the vanishing flat-space 3-divergence (21) of the 3vector (22). For that purpose, we expand equation (19) as

$$
\partial_{\alpha}\left[(\epsilon+p) u^{\alpha}\right]+(\epsilon+p) \Gamma_{\beta \alpha}^{\beta} u^{\alpha}=u^{\alpha} \partial_{\alpha} p,
$$

where $\Gamma_{\beta \gamma}^{\alpha}=g^{\alpha \delta} \Gamma_{\delta \beta \gamma}$ are the common 4-dimensional Christoffel symbols of the second kind. Because of [13]

$$
\Gamma_{\beta \alpha}^{\beta}=\partial_{\alpha} \ln \sqrt{-g},
$$

with the determinant $g=\operatorname{det} g_{\alpha \beta}$, and our assumptions of stationarity and axisymmetry all temporal and azimuthal derivatives $\partial_{t} \ldots$ and $\partial_{\phi} \ldots$ vanish. Hence, equation $(\mathrm{C} 1)$ becomes

$$
\partial_{m}\left[(\epsilon+p) u^{m}\right]+(\epsilon+p) u^{m} \partial_{m} \ln \sqrt{-g}=u^{m} \partial_{m} p .
$$

To compute the determinant $g$, we use its relation to the determinant $k=\operatorname{det} k_{m n}$ (see equation (2.27) of Gourgoulhon et al. [6]), the fact that in MTCMA coordinates (Sect. II B) $k=A^{4} r^{2}$, and the definitions (2) and (3):

$$
\sqrt{-g}=N M \sqrt{k}=e^{2 \alpha+\gamma} r^{2} \sin \theta .
$$

For the right hand side of equation $(\mathrm{C} 2)$, we apply the temporal component

$$
u^{m} \partial_{m} p=-(\epsilon+p) u^{m} \partial_{m} \ln u_{t}
$$

of the general relativistic Euler equation (25), obtained by setting $\alpha=t$ in that equation and using stationarity. Hence, equation (C2) becomes

$$
\begin{aligned}
& \partial_{m}\left[e^{2 \alpha+\gamma}(\epsilon+p) u_{t} u^{m}\right] \\
&+e^{2 \alpha+\gamma}(\epsilon+p) u_{t}\left(\frac{2}{r} u^{r}+\cot \theta u^{\theta}\right)=0
\end{aligned}
$$

which is equal to equation (21). 


\section{Appendix D: Meridional components}

Let us consider the general relativistic Euler equation (20) rewritten in the form of equation (26). This appendix deals with the meridional components of that equation, which means setting $a=m$ in equation (26) such that

$$
\frac{\partial_{m} p}{\epsilon+p}=\frac{1}{2} u^{\alpha} u^{\beta} \partial_{m} g_{\alpha \beta}+u_{t} u^{n} \partial_{n} l_{m}
$$

For the second term on the right hand side, we write

$$
u^{n} \partial_{n} l_{m}=r w\left(\begin{array}{c}
u^{\theta} \\
-u^{r}
\end{array}\right)+u^{n} \partial_{m} l_{n},
$$

with

$$
w=\frac{1}{r}\left(\partial_{\theta} l_{r}-\partial_{r} l_{\theta}\right),
$$

begin the quantity ' $\omega$ ' defined in Eriguchi et al. [3] in the Newtonian limit. Then, using the constraint (7) a short calculation shows that

$$
\frac{1}{2} u^{\alpha} u^{\beta} \partial_{m} g_{\alpha \beta}-u_{t} u^{n} \partial_{m} \frac{u_{n}}{u_{t}}=-\partial_{m} \ln u_{t}+u_{t} u^{\phi} \partial_{m} \frac{u_{\phi}}{u_{t}} .
$$

Hence, equation (D1) can be brought in the form (29), which has a form similar to equations (7) and (8) of Eriguchi et al. [3].

\section{Appendix E: Special Laplacian}

In the following, we show that there is a Laplacian hidden behind the quantity $w$ defined in equation (D2). For that purpose, we extend equations (15) and (17) of Eriguchi et al. [3] to general relativity.

The first step is to define the quantity

$$
D=\partial_{r}^{2} \psi+\frac{\sin \theta}{r^{2}} \partial_{\theta}\left(\frac{1}{\sin \theta} \partial_{\theta} \psi\right),
$$

which is equal to the left hand side of equation (15) of Eriguchi et al. [3]. Then, equation (24) allows us to write

$$
D=\sin \theta\left[-\partial_{r}\left(r^{2} \varrho u^{\theta}\right)+\partial_{\theta}\left(\varrho u^{r}\right)\right] .
$$

Next, we use $u^{m}=g^{m \alpha} u_{\alpha}$, and the decompositions (2.9b) and (2.25b) of Gourgoulhon et al. [6] to obtain after a short computation

$$
u^{m}=u_{t} k^{m n}\left(\frac{c_{n}}{\tilde{\varrho}}-l_{n}\right),
$$

with

$$
\begin{aligned}
c_{m} & =\frac{\tilde{\varrho}}{u_{t}} k_{m n}\left[\frac{M^{n}}{M^{2}}\left(M^{p} u_{p}+u_{\phi}\right)-\frac{N^{n} N^{a}}{N^{2}} u_{a}-\frac{N^{n}}{N^{2}} u_{t}\right], \\
\varrho & =\frac{\varrho u_{t}}{A^{2}} .
\end{aligned}
$$

The only non-vanishing components of the 2-metric $k^{m n}$ are $k^{r r}=1 / A^{2}$ and $k^{\theta \theta}=1 /\left(A^{2} r^{2}\right)$. Therefore, equation (E2) gives

$$
\begin{aligned}
\partial_{r}\left(r^{2} \varrho u^{\theta}\right) & =\partial_{r} c_{\theta}-\partial_{r}\left(\varrho l_{\theta}\right), \\
\partial_{\theta}\left(\varrho u^{r}\right) & =\partial_{\theta} c_{r}-\partial_{\theta}\left(\varrho l_{r}\right) .
\end{aligned}
$$

By inverting equation (E2) to

$$
l_{m}=\frac{1}{u_{t}}\left(\frac{u_{t}}{\tilde{\varrho}} c_{m}-k_{m n} u^{n}\right)
$$

and using equation (24) we also find

$$
\begin{aligned}
& l_{r}=\frac{1}{\tilde{\varrho}}\left(c_{r}-\frac{\partial_{\theta} \psi}{r^{2} \sin \theta}\right), \\
& l_{\theta}=\frac{1}{\tilde{\varrho}}\left(c_{\theta}+\frac{\partial_{r} \psi}{\sin \theta}\right) .
\end{aligned}
$$

Now we combine equations (D2) and (E3-E6) such that equation (E1) becomes

$$
\begin{aligned}
D= & \sin \theta\left(\partial_{\theta} c_{r}-\partial_{r} c_{\theta}\right)-\tilde{\varrho} r \sin \theta w \\
& +\left[\left(\partial_{r} \psi+c_{\theta} \sin \theta\right) \partial_{r}+\left(\frac{\partial_{\theta} \psi}{r^{2}}-c_{r} \sin \theta\right) \partial_{\theta}\right] \ln \tilde{\varrho},
\end{aligned}
$$

which extends equation (15) of Eriguchi et al. [3] to general relativity.

Next, we introduce the quantity [3]

$$
\chi=\frac{\psi \cos \phi}{r \sin \theta}
$$

to write equation (E7) in the form

$$
\begin{aligned}
D= & \frac{r \sin \theta}{\cos \phi}\left\{\frac{\cos \phi}{r}\left(\partial_{\theta} c_{r}-\partial_{r} c_{\theta}\right)-\tilde{\varrho} w \cos \phi\right. \\
& +\left[\left(\partial_{r} \chi+\frac{\chi}{r}+\frac{c_{\theta}}{r} \cos \phi\right) \partial_{r}\right. \\
& \left.\left.+\frac{1}{r^{2}}\left(\partial_{\theta} \chi+\chi \cot \theta-r c_{r} \cos \phi\right) \partial_{\theta}\right] \ln \varrho\right\} .
\end{aligned}
$$

In the Newtonian limit, the expression in curly brackets becomes the right hand side of equation (17) of Eriguchi et al. [3], i.e. equation (33) extends equation (17) of Eriguchi et al. [3] to general relativity under condition (32).

\section{Appendix F: Integral expansion}

Below, we expand the four integrals appearing in Sect. III B in terms of trigonometric functions and Legendre polynomials.

\section{2-scalar}

For the integral (49) we use the expansion

$$
\begin{aligned}
\ln \left|\vec{x}-\vec{x}^{\prime}\right|= & \ln \max \left(r, r^{\prime}\right)-\sum_{l=1}^{\infty} \frac{1}{l} \frac{\min ^{l}\left(r, r^{\prime}\right)}{\max ^{l}\left(r, r^{\prime}\right)} \\
& \cdot\left(\cos (l \theta) \cos \left(l \theta^{\prime}\right)+\sin (l \theta) \sin \left(l \theta^{\prime}\right)\right)
\end{aligned}
$$


of Komatsu et al. [5]. Applying the von Neumann boundary condition

$$
S(r, \pi+\theta)=S(r, \pi-\theta),
$$

a short computation shows that

$$
\begin{aligned}
& \Phi(r, \theta)= \frac{1}{\pi} \int_{0}^{\infty} \mathrm{d} r^{\prime} r^{\prime} \ln \max \left(r, r^{\prime}\right) \int_{0}^{\pi} \mathrm{d} \theta^{\prime} S\left(r^{\prime}, \theta^{\prime}\right) \\
&-\frac{1}{\pi} \sum_{l=1}^{\infty} \frac{1}{l} \cos (l \theta) \int_{0}^{\infty} \mathrm{d} r^{\prime} r^{\prime} \frac{\min ^{l}\left(r, r^{\prime}\right)}{\max ^{l}\left(r, r^{\prime}\right)} \\
& \cdot \int_{0}^{\pi} \mathrm{d} \theta^{\prime} \cos \left(l \theta^{\prime}\right) S\left(r^{\prime}, \theta^{\prime}\right) .
\end{aligned}
$$

We use this expansion for the basic geometry field $\alpha$, i.e. equation (40). In that case, the potential is $\Phi=\alpha+\nu$ and the source $S=S_{\alpha}$.

For the basic geometry field $\beta$, i.e. equation (41), the integral (49) has to be evaluated. The potential is $\Phi=$ $r \sin \theta(\beta+\nu)$, which has to vanish on the rotation axis. Therefore, we apply the Dirichlet boundary condition

$$
S(r, \pi+\theta)=-S(r, \pi-\theta)
$$

and find

$$
\begin{array}{r}
\Phi(r, \theta)=-\frac{1}{\pi} \sum_{l=1}^{\infty} \frac{1}{l} \sin (l \theta) \int_{0}^{\infty} \mathrm{d} r^{\prime} r^{\prime} \frac{\min ^{l}\left(r, r^{\prime}\right)}{\max ^{l}\left(r, r^{\prime}\right)} \\
\cdot \int_{0}^{\pi} \mathrm{d} \theta^{\prime} \sin \left(l \theta^{\prime}\right) S\left(r^{\prime}, \theta^{\prime}\right),
\end{array}
$$

with the source $S=S_{\beta}$.

\section{3-scalar}

For the integral (50) we use the expansion [5]

$$
\begin{aligned}
\frac{1}{\left|\vec{x}-\vec{x}^{\prime}\right|}=\sum_{l=0}^{\infty} \sum_{m=-l}^{l} \frac{(l-m) !}{(l+m) !} \frac{\min ^{l}\left(r, r^{\prime}\right)}{\max ^{l+1}\left(r, r^{\prime}\right)} \\
\cdot P_{l}^{m}\left(\cos \theta^{\prime}\right) P_{l}^{m}(\cos \theta) e^{i m\left(\phi-\phi^{\prime}\right)},
\end{aligned}
$$

where $P_{l}^{m}$ are the associated Legendre polynomials. In case of an axisymmetric source

$$
S(\vec{x})=S(r, \theta),
$$

it is easy to show that

$$
\begin{array}{r}
\Phi(r, \theta)=-\frac{1}{2} \sum_{l=0}^{\infty} P_{l}(\cos \theta) \int_{0}^{\infty} \mathrm{d} r^{\prime} r^{\prime 2} \frac{\min ^{l}\left(r, r^{\prime}\right)}{\max ^{l+1}\left(r, r^{\prime}\right)} \\
\cdot \int_{0}^{\pi} \mathrm{d} \theta^{\prime} P_{l}\left(\cos \theta^{\prime}\right) \sin \theta^{\prime} S\left(r^{\prime}, \theta^{\prime}\right) .
\end{array}
$$

We apply this result to equation (39), and choose the potential $\Phi=\nu$ together with the source $S=S_{\nu}$.
The integral (50) also appears in the equation for the basic matter field $\chi_{0}(44)$. In that case, the source is no longer axisymmetric but obeys

$$
S(\vec{x})=S(r, \theta) \cos \phi .
$$

Then, it is possible to show that

$$
\begin{aligned}
\Phi(r, \theta, \phi) & =\Phi(r, \theta) \cos \phi \\
\Phi(r, \theta) & =\Phi_{0}(r, \theta)+\ldots
\end{aligned}
$$

with

$$
\begin{aligned}
\Phi_{0}(r, \theta)=-\frac{1}{2} \sum_{l=1}^{\infty} & \frac{1}{l(l+1)} P_{l}^{1}(\cos \theta) \\
& \cdot \int_{0}^{\infty} \mathrm{d} r^{\prime} r^{\prime 2} \frac{\min ^{l}\left(r, r^{\prime}\right)}{\max ^{l+1}\left(r, r^{\prime}\right)} \\
& \cdot \int_{0}^{\pi} \mathrm{d} \theta^{\prime} P_{l}^{1}\left(\cos \theta^{\prime}\right) \sin \theta^{\prime} S\left(r^{\prime}, \theta^{\prime}\right) .
\end{aligned}
$$

The dots appearing in equation (F3) serve the same purpose as in equation (50), i.e. they represent a remaining degree of freedom for the choice of the boundary condition of the field $\chi_{0}$. Looking at equation (19) of Eriguchi et al. [3] we actually see that

$$
\Phi(r, \theta)=\Phi_{0}(r, \theta)+\sum_{l=1}^{\infty} a_{l} r^{l} P_{l}^{1}(\cos \theta),
$$

with arbitrary coefficients $a_{l}$. We have to choose these coefficients in such a manner that there is no flow across the surface of the neutron star. For that purpose, we consider surface-adapted coordinates $(\tilde{r}, \tilde{\theta})$ (Sect. III A), where the radial coordinate of the neutron star's surface becomes $\tilde{r}=1$ (37). The condition for no flow across the surface is

$$
\tilde{u}^{r} \stackrel{S}{=} 0
$$

(the letter ' $\mathrm{S}$ ' denotes that this relation holds only on the surface). Using equation (24), it is straightforward to show that this leads to

$$
\partial_{\tilde{\theta}} \psi \stackrel{S}{=} 0
$$

As

$$
\psi=r \sin \theta \chi_{0}=\tilde{r} R(\tilde{\theta}) \sin \tilde{\theta} \chi_{0}
$$

we then find

$$
\left(1+\frac{R^{\prime}(\tilde{\theta})}{R(\tilde{\theta})} \tan \tilde{\theta}\right) \chi_{0}+\tan \tilde{\theta} \partial_{\tilde{\theta}} \chi_{0} \stackrel{S}{=} 0
$$

Though not being the only mathematical solution, we currently limit ourselves to the case

$$
\chi_{0} \stackrel{S}{=} 0
$$


which is the Dirichlet boundary condition and which leads to

$$
\partial_{\tilde{\theta}} \chi_{0} \stackrel{S}{=} 0
$$

because in surface-adapted coordinates the surface corresponds to a constant radial coordinate $\tilde{r}$. This shows that the Dirichlet boundary condition (F7) implies the constraint (F6) for no flow across the surface. In terms of the potential

$$
\tilde{\Phi}(\tilde{r}, \tilde{\theta})=\Phi(r, \theta)
$$

equation (F7) implies

$$
\tilde{\Phi}(1, \tilde{\theta})=0
$$

For this boundary condition, it can be shown that

$$
\begin{aligned}
\tilde{\Phi}(\tilde{r}, \tilde{\theta})=\tilde{\Phi}_{0}(\tilde{r}, \tilde{\theta}) & -\frac{1}{2} \sum_{l=1}^{\infty} \frac{2 l+1}{l(l+1)} P_{l}^{1}(\cos \tilde{\theta}) \tilde{r}^{l} \quad(\mathrm{~F} 8) \\
& \cdot \int_{0}^{\pi} \mathrm{d} \tilde{\theta}^{\prime} P_{l}^{1}\left(\cos \tilde{\theta}^{\prime}\right) \sin \tilde{\theta}^{\prime} \tilde{\Phi}_{0}\left(1, \tilde{\theta}^{\prime}\right),
\end{aligned}
$$

with

$$
\tilde{\Phi}_{0}(\tilde{r}, \tilde{\theta})=\Phi_{0}(r, \theta)
$$

Note that we use the expansions (F4) and (F8) both for the potential $\Phi(r, \theta)=\chi_{0}(r, \theta)$ and the source $S(r, \theta)=$ $S_{\chi_{0}}(r, \theta)$.

\section{2-vector}

Next, we consider the third integral of Sect. III B to evaluate the potential $\Phi^{m}=e^{2(\alpha+\nu)} M^{m}$ and the source $S^{m}=S_{M}^{m}$. Similar to Sect. F1, we have to specify boundary conditions. We use the 2-dimensional Cartesian coordinates introduced in equation (51), in which the potential and the source have the components $\left(\Phi^{x}, \Phi^{z}\right)$ and $\left(S^{x}, S^{z}\right)$, respectively. We apply the Dirichlet boundary condition on the $x$-component $\Phi^{x}$, and the von Neumann one on the $z$-component $\Phi^{z}$. These two quantities are then governed by equations resulting from replacing

$$
\begin{aligned}
\Phi(r, \theta) & \rightarrow \Phi^{x}(r, \theta), \\
S\left(r^{\prime}, \theta^{\prime}\right) & \rightarrow S^{x}\left(r^{\prime}, \theta^{\prime}\right)
\end{aligned}
$$

in equation (F2) and

$$
\begin{aligned}
\Phi(r, \theta) & \rightarrow \Phi^{z}(r, \theta), \\
S\left(r^{\prime}, \theta^{\prime}\right) & \rightarrow S^{z}\left(r^{\prime}, \theta^{\prime}\right)
\end{aligned}
$$

in equation (F1).

\section{3-vector}

The fourth integral of Sect. III B is used to compute the potential $\Phi^{a}=N^{a}$ and the source $S^{a}=S_{N}^{a}$. In this case, the derivation of the expansion in terms of Legendre polynomials is somewhat lengthy, but still straightforward such that we give only the result:

$$
\begin{aligned}
& \Phi^{a}(r, \theta)=-\frac{1}{2}\left(\begin{array}{cc}
\sin \theta & 0 \\
\frac{\cos \theta}{r} & 0 \\
0 & \frac{1}{r \sin \theta}
\end{array}\right) \sum_{l=1}^{\infty} \frac{1}{l(l+1)} P_{l}^{1}(\cos \theta) \\
& \int_{0}^{\infty} \mathrm{d} r^{\prime} r^{\prime 2} \frac{\min ^{l}\left(r, r^{\prime}\right)}{\max ^{l+1}\left(r, r^{\prime}\right)} \\
& \int_{0}^{\pi} \mathrm{d} \theta^{\prime} P_{l}^{1}\left(\cos \theta^{\prime}\right) \sin \theta^{\prime} \\
& \cdot\left(\begin{array}{c}
\sin \theta^{\prime} S^{r}\left(r^{\prime}, \theta^{\prime}\right)+r^{\prime} \cos \theta^{\prime} S^{\theta}\left(r^{\prime}, \theta^{\prime}\right) \\
r^{\prime} \sin \theta^{\prime} S^{\phi}\left(r^{\prime}, \theta^{\prime}\right)
\end{array}\right)^{T} \\
& -\frac{1}{2}\left(\begin{array}{c}
\cos \theta \\
-\frac{\sin \theta}{r} \\
0
\end{array}\right) \sum_{l=0}^{\infty} P_{l}(\cos \theta) \\
& \cdot \int_{0}^{\infty} \mathrm{d} r^{\prime} r^{\prime 2} \frac{\min ^{l}\left(r, r^{\prime}\right)}{\max ^{l+1}\left(r, r^{\prime}\right)} \\
& \cdot \int_{0}^{\pi} \mathrm{d} \theta^{\prime} P_{l}\left(\cos \theta^{\prime}\right) \sin \theta^{\prime} \\
& \cdot\left(\cos \theta^{\prime} S^{r}\left(r^{\prime}, \theta^{\prime}\right)-r^{\prime} \sin \theta^{\prime} S^{\theta}\left(r^{\prime}, \theta^{\prime}\right)\right) \text {. }
\end{aligned}
$$

\section{Appendix G: Tests}

Here, we present convergence and consistency tests performed with GRNS. For that purpose, we introduce the convergence indicator

$$
C_{s}=100 \max _{F} \frac{\sum_{\text {grid }}\left|F_{s}-F_{s-1}\right|}{\sum_{\text {grid }}\left|F_{s}\right|},
$$

where $F_{s}$ is the distribution of the basic field $F$ at the iteration step $s$, and t The sums extend over the numerical grid. First the fraction is evaluated for all basic fields $F$, and then the maximum value determines the convergence indicator $C_{s}$. That way, this quantity is most sensitive to the basic field which converges least. Note that the convergence indicator becomes $C_{s}=0$ for perfect convergence. Fig. 9 shows convergence tests for three different resolutions. We see that for the fundamental mode GRNS converges for all three resolutions, i.e. the convergence indicator $C_{s}$ approaches zero during the fixed point iteration. For higher modes, the convergence indicator drops initially but then starts to fluctuate, never getting close to the value zero. Improving the weight function given in equation (56) might improve this behavior. We have also considered different numbers of terms $l_{\max }$ used in the sums (57), namely $3,10,50$, which 


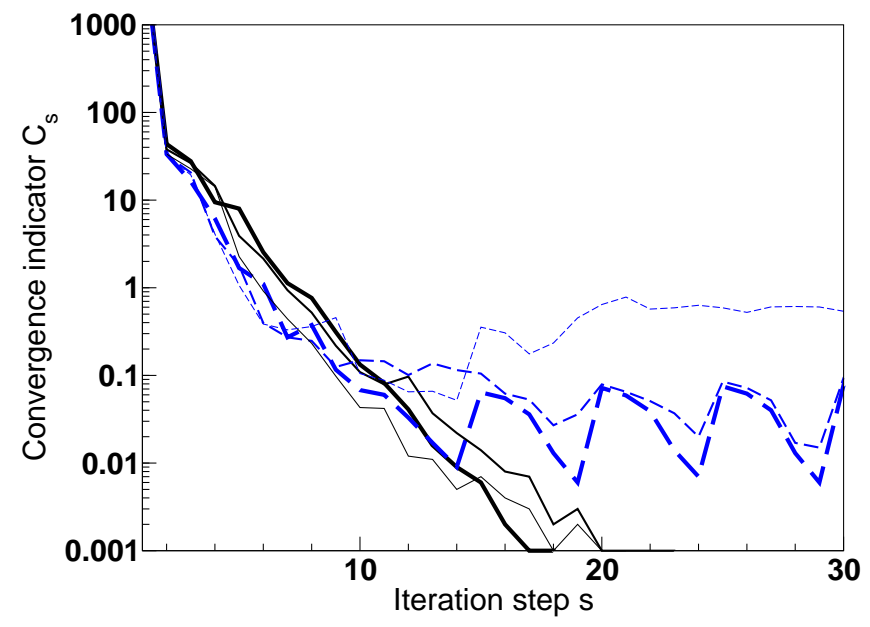

FIG. 9: Convergence tests. Dependence of the convergence indicator $C_{s}$ defined in equation (G1) on the iteration step $s$ for a model similar to the reference model. The three solid lines refer to grid resolutions of $59 \times 52,150 \times 156$, and $501 \times 507$ zones (the higher the resolution, the thinner the corresponding line). The blue dashed lines refer to the first higher mode.

results is a convergence behavior similar to that shown in Fig. 9, when the three grid resolutions are replaced with the three values of $l_{\max }$. Eventually, we have validated the correct behavior of GRNS, when the spatial extension of the numerical grid is changed.

\section{Appendix H: Outlook on the magnetic field}

If a magnetic field is included, the stress-energy tensor (6) has the additional contribution

$$
T_{\alpha \beta}^{\mathrm{EM}}=F_{\alpha \gamma} F_{\beta}^{\gamma}-\frac{1}{4} g_{\alpha \beta} F_{\gamma \delta} F^{\gamma \delta},
$$

where $F_{\alpha \beta}=\partial_{\alpha} A_{\beta}-\partial_{\beta} A_{\alpha}$ is the electromagnetic field strength expressed in terms of the electromagnetic 4vector potential $A_{\alpha}$. Moreover, we do not only have to solve Einstein's field equation (11), but also Maxwell's field equation

$$
\nabla_{\alpha} F^{\alpha \beta}=\rho_{q} u^{\beta}
$$

where $\rho_{q}$ is the charge density. A lengthy, but straightforward computation shows that the general relativistic Euler equation (20) becomes

$$
(\epsilon+p) u^{\beta} \nabla_{\beta} u^{\alpha}=-q^{\alpha \beta} \nabla_{\beta} p-\rho_{q} F_{\beta}^{\alpha} u^{\beta},
$$

and equation (27) changes to

$$
u^{m} \partial_{m} l_{\phi}=-\frac{\rho_{q} u^{m}\left(l_{\phi} \partial_{m} A_{t}+\partial_{m} A_{\phi}\right)}{u_{t}(\epsilon+p)}
$$

Hence, the simple solution method (28) does no longer work if a magnetic field is present.

\section{Appendix I: Sources}

In Sect. II C, we have written the covariant Poisson equations (B3-B7) of Gourgoulhon et al. [6] to flat-space Poisson equations. The sources $S_{\nu}, S_{\alpha}, S_{\beta}, S_{M}^{m}$, and $S_{N}^{a}$ of these equations, and the source $S_{\chi_{0}}$ of the Poisson equation (33) are listed in the following. Similar to Appendix A, several new mathematical quantities appear below, which are defined in Gourgoulhon et al. [6].

The scalar sources are

$$
\begin{aligned}
S_{\nu}=A^{2}\{ & 4 \pi\left(E+S_{a}^{a}\right)+K_{a b} K^{a b}+\frac{L^{2}}{2}-\left[\frac{1}{A^{2}}+\left(m^{r}\right)^{2}\right]\left(\nu_{, r}\right)^{2}-\left[\frac{1}{(r A)^{2}}+\left(m^{\theta}\right)^{2}\right]\left(\nu_{, \theta}\right)^{2}-\left(m^{r}\right)^{2} \nu_{, r r}-\left(m^{\theta}\right)^{2} \nu_{, \theta \theta} \\
& \left.-2 m^{r} m^{\theta} \nu_{, r \theta}-\left(m^{r} m_{, r}^{r}+m^{\theta} m_{, \theta}^{r}\right) \nu_{, r}-\left(m^{r} m_{, r}^{\theta}+m^{\theta} m^{\theta}, \theta\right) \nu_{, \theta}-2 m^{r} m^{\theta} \nu_{, r} \nu_{, \theta}\right\}-\beta_{, r} \nu_{, r}-\frac{\beta_{, \theta} \nu_{, \theta}}{r^{2}}
\end{aligned}
$$

and

$$
\begin{gathered}
S_{\alpha}=A^{2}\left\{8 \pi s+\frac{1}{N}\left[\left(q^{r}+\omega m^{r}\right) \kappa_{, r}+\left(q^{\theta}+\omega m^{\theta}\right) \kappa_{, \theta}\right]+\frac{2}{M N}\left[\kappa_{r}[M, q]^{r}+\kappa_{\theta}[M, q]^{\theta}\right]+3 \kappa_{m} \kappa^{m}\right. \\
\left.+\frac{1}{2}\left(\kappa_{m n} \kappa^{m n}+\kappa^{2}+L_{m n} L^{m n}\right)\right\}-\left(\nu_{, r}\right)^{2}-\left(\frac{\nu_{, \theta}}{r}\right)^{2}
\end{gathered}
$$

and

$$
\begin{aligned}
S_{\beta}=\frac{A^{2}}{e^{\gamma}}\{ & 8 \pi M N s_{m}^{m}-2 \kappa_{r}[M, q]^{r}-2 \kappa_{\theta}[M, q]^{\theta}-M\left(q^{r}+\omega m^{r}\right) \kappa_{, r}-M\left(q^{\theta}+\omega m^{\theta}\right) \kappa_{, \theta} \\
+ & \left.M N\left(\kappa_{m n} \kappa^{m n}+\kappa^{2}-L_{m n} L^{m n}\right)\right\}-r \sin \theta\left[\left(\gamma_{r}\right)^{2}+\frac{1}{r^{2}}(\gamma, \theta)^{2}\right]
\end{aligned}
$$


and

$$
S_{\chi_{0}}=\frac{1}{r}\left(\partial_{\theta} c_{r}-\partial_{r} c_{\theta}\right)-\varrho \varrho \varrho r s i n \theta\left(\frac{f(\psi)}{u_{t}}-u^{\phi} L^{\prime}(\psi)\right)+\left[\left(\partial_{r} \chi_{0}+\frac{\chi_{0}}{r}+\frac{c_{\theta}}{r}\right) \partial_{r}+\frac{1}{r^{2}}\left(\partial_{\theta} \chi_{0}+\cot \theta \chi_{0}-r c_{r}\right)\right] \partial_{\theta} \ln \tilde{\varrho} .
$$

The two components of the 2-vector source $S_{M}^{m}$ are the $r$-component

$$
\begin{aligned}
S_{M}^{r}=A^{2} N^{2} & \left\{4(\alpha+\nu)_{, r}\left[M^{r}(\alpha+\nu)_{, r}+M_{, r}^{r}\right]+\frac{4}{r^{2}}(\alpha+\nu)_{, \theta}\left[M^{r}(\alpha+\nu)_{, \theta}+M_{, \theta}^{r}\right]\right. \\
& \left.-\frac{4}{r} M^{\theta}(\alpha+\nu)_{, \theta}+{S^{\prime}}^{\prime r}+2 M^{r} S_{\alpha}\right\}
\end{aligned}
$$

and the $\theta$-component

$$
\begin{aligned}
S_{M}^{\theta}=A^{2} N^{2}\{ & 4(\alpha+\nu)_{, r}\left[M^{\theta}(\alpha+\nu)_{, r}+M_{, r}^{\theta}\right]+\frac{4}{r^{2}}(\alpha+\nu)_{, \theta}\left[M^{\theta}(\alpha+\nu)_{, \theta}+M_{, \theta}^{\theta}\right] \\
& \left.+\frac{4}{r} M^{\theta}(\alpha+\nu)_{, r}+\frac{4}{r^{3}} M^{r}(\alpha+\nu)_{, \theta}+{S^{\prime}}^{\theta}{ }_{M}+2 M^{\theta} S_{\alpha}\right\} .
\end{aligned}
$$

The three components of the 3 -vector source $S_{N}^{a}$ are the $r$-component

$$
\begin{aligned}
& S_{N}^{r}=A^{2}\left\{-16 \pi N J^{r}-2 K^{r r} N_{, r}-2 K^{r \theta} N_{, \theta}-2 m^{r} m^{\theta} N_{, r \theta}^{r}-N_{, r}^{r}\left\{2\left[\frac{1}{A^{2}}+\left(m^{r}\right)^{2}\right] \alpha_{, r}+m^{r} m^{\theta}\left(2 \alpha_{, \theta}-\mu, \theta\right)\right.\right. \\
& \left.+m^{r} \frac{M_{, \theta}^{\theta}}{M}+m^{\theta} \frac{M_{, \theta}^{r}}{M}\right\}-N_{, \theta}^{r}\left\{2\left[\frac{1}{(r A)^{2}}+2\left(m^{\theta}\right)^{2}\right] \alpha_{, \theta}+m^{r} m^{\theta}\left(\mu_{, r}+4 \alpha_{, r}+\frac{1}{r}\right)-\frac{m^{r} M_{, \theta}^{r}}{r^{2} M}\right. \\
& \left.+\frac{m^{\theta}}{M}\left(M_{, r}^{r}+2 M_{, \theta}^{\theta}\right)\right\}-2 N_{, r}^{\theta}\left[\frac{\alpha_{, \theta}}{A^{2}}+\left(m^{r}\right)^{2} \mu_{, \theta}-m^{r} \frac{M_{, \theta}^{r}}{M}\right]-N_{, \theta}^{\theta}\left\{-2\left[\frac{1}{A^{2}}+\left(m^{r}\right)^{2}\right] \alpha_{, r}\right. \\
& \left.+2 m^{r} m^{\theta}\left(\mu_{, \theta}-\alpha_{, \theta}\right)-2 m^{r} \frac{M_{, \theta}^{\theta}}{M}+\frac{m^{\theta}}{M}\left(r^{2} M_{, r}^{\theta}-M_{, \theta}^{r}\right)\right\}-N_{, \theta}^{\varphi}\left\{\frac{2 M^{r}}{(r A)^{2}}\left(\mu_{, \theta}-\alpha_{, \theta}\right)+2 \frac{M^{\theta}}{A^{2}}\left[\alpha_{, r}-\mu_{, r}\right.\right. \\
& \left.+\frac{1}{r}+\frac{\left(A m^{r}\right)^{2}}{r}\right]+2 m^{r} m^{\theta}\left(M_{, \theta}^{\theta}-M_{, r}^{r}\right)-\frac{1}{r^{2}}\left[\frac{1}{A^{2}}-\left(m^{r}\right)^{2}+\left(r m^{\theta}\right)^{2}\right] M_{, \theta}^{r}+\left[\frac{1}{A^{2}}+\left(m^{r}\right)^{2}\right. \\
& \left.\left.-\left(r m^{\theta}\right)^{2}\right] M_{, r}^{\theta}\right\}-N^{r}\left\{2\left[\frac{1}{A^{2}}+2\left(m^{r}\right)^{2}\right] \alpha_{, r}\left(\mu_{, r}-\frac{1}{r}\right)-\left[\frac{1}{A^{2}}-\left(m^{r}\right)^{2}\right] \frac{M_{, r r}}{M}+m^{r} m^{\theta}\left(-\mu_{, r} \mu_{, \theta}\right.\right. \\
& \left.+4 \alpha_{, \theta} \mu_{, r}-2 \frac{\alpha_{, \theta}}{r}-2 \alpha_{, r} \alpha_{, \theta}+\frac{M_{, r \theta}}{M}-2 \frac{A_{, r \theta}}{A}\right)+2\left(m^{r}\right)^{2}\left[\frac{\mu_{, r}}{r}-\left(\alpha_{, r}\right)^{2}-\frac{A_{, r r}}{A}\right]+\frac{m^{r}}{M}\left[2 M _ { , r } ^ { r } \left(\mu_{, r}-4 \alpha_{, r}\right.\right. \\
& \left.\left.-\frac{1}{r}\right)-M_{, r}^{\theta}\left(\mu_{, \theta}+2 \alpha_{, \theta}\right)+2 M_{, \theta}^{\theta}\left(\mu_{, r}-\alpha_{, r}-\frac{1}{r}\right)-2 M_{, r r}^{r}-M_{, r \theta}^{\theta}\right]+\frac{m^{\theta}}{M}\left[M_{, r}^{r}\left(\mu_{, \theta}-4 \alpha_{, \theta}\right)\right. \\
& \left.\left.-M_{, r \theta}^{r}\right]-\frac{1}{M^{2}}\left[2\left(M_{, r}^{r}\right)^{2}+M_{, r}^{r} M_{, \theta}^{\theta}+r^{2}\left(M_{, r}^{\theta}\right)^{2}\right]\right\}-N^{\theta}\left\{\frac{2}{A^{2}} \alpha_{, \theta} \mu_{, r}+m^{r} m^{\theta}\left[4 \alpha_{, \theta} \mu_{, \theta}-2\left(\alpha_{, \theta}\right)^{2}\right.\right. \\
& \left.-\left(\mu_{, \theta}\right)^{2}+\frac{M_{, \theta \theta}}{M}-2 \frac{A_{, \theta \theta}}{A}\right]+2\left(m^{r}\right)^{2}\left(\frac{\mu_{, \theta}}{r}-\frac{\alpha_{, \theta}}{r}-\alpha_{, r} \alpha_{, \theta}+2 \mu_{, \theta} \alpha_{, r}-\frac{A_{, r \theta}}{A}\right)+\frac{m^{r}}{M}\left[2 M_{, r}^{r}\left(\mu_{, \theta}-\alpha_{, \theta}\right)\right. \\
& \left.-2 M_{, \theta}^{r}\left(3 \alpha_{, r}+\frac{1}{r}\right)+M_{, \theta}^{\theta}\left(\mu_{, \theta}-4 \alpha_{, \theta}\right)-M_{, \theta \theta}^{\theta}-2 M_{, r \theta}^{r}\right]+\frac{m^{\theta}}{M}\left[M_{, \theta}^{r}\left(\mu_{, \theta}-4 \alpha_{, \theta}\right)-M_{, \theta \theta}^{r}\right] \\
& \left.-\frac{1}{M^{2}}\left(M_{, \theta}^{r} M_{, \theta}^{\theta}+2 M_{, r}^{r} M_{, \theta}^{r}+r^{2} M_{, r}^{\theta} M_{, \theta}^{\theta}\right)\right\}-\left(m^{r}\right)^{2}\left\{N_{, r r}^{r}+\left(\frac{1}{r}+\mu_{, r}\right) N_{, r}^{r}-\left[\frac{1}{r^{2}}+\left(\mu_{, r}\right)^{2}\right] N^{r}\right\} \\
& \left.-\left(m^{\theta}\right)^{2} N_{, \theta \theta}^{r}+\left(m^{\theta}\right)^{2} \mu_{, \theta} N_{, \theta}^{r}+\frac{2}{r}\left(m^{r}\right)^{2} N_{, \theta}^{\theta}-\left(m^{r}\right)^{2} \beta_{, r \theta} N^{\theta}\right\} \\
& -\beta_{, r} N_{, r}^{r}+\left[\frac{2}{r} \beta_{, r}+\left(\beta_{, r}\right)^{2}\right] N^{r}-\frac{1}{r^{2}} \beta_{, \theta} N_{, \theta}^{r}+\left(\frac{2}{r} \beta_{, \theta}+2 \cot \theta \beta_{, r}+\beta_{, r \theta}+2 \beta_{, r} \beta_{, \theta}\right) N^{\theta},
\end{aligned}
$$

the $\theta$-component

$$
S_{N}^{\theta}=A^{2}\left\{-16 \pi N J^{\theta}-2 K^{\theta r} N_{, r}-2 K^{\theta \theta} N_{, \theta}-2 m^{r} m^{\theta} N_{, r \theta}^{\theta}-N_{, r}^{r}\left\{-2\left[\frac{1}{(r A)^{2}}+\left(m^{\theta}\right)^{2}\right] \alpha_{, \theta}+2 m^{r} m^{\theta}\left(\mu_{, r}-\alpha_{, r}\right)\right.\right.
$$




$$
\begin{aligned}
& \left.+\frac{1}{M}\left[m^{r}\left(\frac{M_{, \theta}^{r}}{r^{2}}-M_{, r}^{\theta}\right)-2 m^{\theta} M_{, r}^{r}\right]\right\}-N_{, \theta}^{r}\left[\frac{2}{(r A)^{2}} \alpha_{, r}+2\left(m^{\theta}\right)^{2} \mu_{, r}-2 m^{\theta} \frac{M_{, r}^{\theta}}{M}\right]-N_{, r}^{\theta}\left\{2 \left[\frac{1}{A^{2}}\right.\right. \\
& \left.\left.+2\left(m^{r}\right)^{2}\right] \alpha_{, r}+m^{r} m^{\theta}\left(\mu_{, \theta}+4 \alpha_{, \theta}\right)+\frac{1}{M}\left[m^{r}\left(2 M_{, r}^{r}+M_{, \theta}^{\theta}\right)-m^{\theta} r^{2} M_{, r}^{\theta}\right]\right\}-N_{, \theta}^{\theta}\left\{2 \left[\frac{1}{(r A)^{2}}\right.\right. \\
& \left.\left.+\left(m^{\theta}\right)^{2}\right] \alpha_{, \theta}+m^{r} m^{\theta}\left(2 \alpha_{, r}-\mu_{, r}+\frac{1}{r}\right)+\frac{1}{M}\left(m^{r} M_{, r}^{\theta}+m^{\theta} M_{, r}^{r}\right)\right\}-N_{, r}^{\varphi}\left\{\frac{2 M^{r}}{(r A)^{2}}\left(\alpha_{, \theta}-\mu_{, \theta}\right)\right. \\
& +2 \frac{M^{\theta}}{A^{2}}\left[\mu_{, r}-\alpha_{, r}-\frac{1}{r}-\frac{\left(A m^{r}\right)^{2}}{r}\right]+2 m^{r} m^{\theta}\left(M_{, r}^{r}-M_{, \theta}^{\theta}\right)+\frac{1}{r^{2}}\left[\frac{1}{A^{2}}-\left(m^{r}\right)^{2}+\left(r m^{\theta}\right)^{2}\right] M_{, \theta}^{r}-\left[\frac{1}{A^{2}}\right. \\
& \left.\left.+\left(m^{r}\right)^{2}-\left(r m^{\theta}\right)^{2}\right] M_{, r}^{\theta}\right\}-N^{r}\left\{-\left(m^{\theta}\right)^{2} \mu_{, r} \mu_{, \theta}+\left(m^{\theta}\right)^{2} \frac{M_{, r \theta}}{M}-\frac{1}{(r A)^{2}}\left(2 \cot \theta \beta_{, r}+\beta_{, r \theta}+2 \beta_{, r} \beta_{, \theta}\right)\right. \\
& +\frac{2}{r}\left[\frac{1}{(r A)^{2}}-\left(m^{\theta}\right)^{2}\right] \alpha_{, \theta}+\frac{2}{(r A)^{2}} \alpha_{, r} \mu_{, \theta}+m^{r} m^{\theta}\left[2 \frac{\mu_{, r}}{r}-4 \frac{\alpha_{, r}}{r}-\frac{1}{r^{2}}+4 \alpha_{, r} \mu_{, r}-2\left(\alpha_{, r}\right)^{2}-\left(\mu_{, r}\right)^{2}+\frac{M_{, r r}}{M}\right. \\
& \left.-2 \frac{A_{, r r}}{A}\right]+2\left(m^{\theta}\right)^{2}\left[\left(2 \mu_{, r}-\alpha_{, r}\right) \alpha_{, \theta}-\frac{A_{, r \theta}}{A}\right]+\frac{m^{r}}{M}\left[\left(\mu_{, r}-4 \alpha_{, r}-\frac{3}{r}\right) M_{, r}^{\theta}-M_{, r r}^{\theta}\right]+\frac{m^{\theta}}{M}\left[M _ { , r } ^ { r } \left(\mu_{, r}\right.\right. \\
& \left.\left.-4 \alpha_{, r}-\frac{1}{r}\right)-6 M_{, r}^{\theta} \alpha_{, \theta}-2 M_{, \theta}^{\theta}\left(-\mu_{, r}+\alpha_{, r}+\frac{1}{r}\right)-M_{, r r}^{r}-2 M_{, r \theta}^{\theta}\right]-\frac{1}{M^{2}}\left(\frac{1}{r^{2}} M_{, r}^{r} M_{, \theta}^{r}\right. \\
& \left.\left.+M_{, r}^{r} M_{, r}^{\theta}+2 M_{, r}^{\theta} M_{, \theta}^{\theta}\right)\right\}-N^{\theta}\left\{2\left[\frac{1}{(r A)^{2}}+2\left(m^{\theta}\right)^{2}\right] \alpha_{, \theta} \mu_{, \theta}+m^{r} m^{\theta}\left[2 \frac{\mu_{, \theta}}{r}-2 \frac{\alpha_{, \theta}}{r}-\mu_{, r} \mu_{, \theta}+4 \alpha_{, r} \mu_{, \theta}\right.\right. \\
& \left.-2 \alpha_{, r} \alpha_{, \theta}+\frac{M_{, r \theta}}{M}-2 \frac{A_{, r \theta}}{A}\right]-2\left(m^{\theta}\right)^{2}\left[\left(\alpha_{, \theta}\right)^{2}+\frac{A_{, \theta \theta}}{A}\right]+\frac{m^{r}}{M}\left[M_{, \theta}^{\theta}\left(\mu_{, r}-4 \alpha_{, r}-\frac{3}{r}\right)-M_{, r \theta}^{\theta}\right] \\
& +\frac{m^{\theta}}{M}\left[2 M_{, r}^{r}\left(\mu, \theta-\alpha_{, \theta}\right)-M_{, \theta}^{r}\left(\mu_{, r}+2 \alpha_{, r}+\frac{1}{r}\right)+2 M_{, \theta}^{\theta}(\mu, \theta-4 \alpha, \theta)-M_{, r \theta}^{r}-2 M_{, \theta \theta}^{\theta}\right] \\
& \left.-\frac{1}{M^{2}}\left[\left(\frac{M_{, \theta}^{r}}{r}\right)^{2}+2\left(M_{, \theta}^{\theta}\right)^{2}+M_{, r}^{r} M_{, \theta}^{\theta}\right]\right\}-\left(m^{r}\right)^{2} N_{, r r}^{\theta}-\frac{1}{A^{2}} \beta_{, r} N_{, r}^{\theta}-\left(m^{r}\right)^{2}\left(\frac{3}{r}-\mu_{, r}\right) N_{, r}^{\theta} \\
& \left.-\left(m^{\theta}\right)^{2}\left(N_{, \theta \theta}^{\theta}+\mu_{, \theta} N_{, \theta}^{\theta}\right)+\left(m^{\theta}\right)^{2}\left(\cot ^{2} \theta+1-\beta_{, \theta \theta}\right) N^{\theta}\right\} \\
& -\frac{1}{r^{2}} \beta_{, \theta} N_{, \theta}^{\theta}+N^{\theta} \frac{1}{r^{2}}\left[4 \cot \theta \beta_{, \theta}+2\left(\beta_{, \theta}\right)^{2}+\beta_{, \theta \theta}\right]
\end{aligned}
$$

and the $\phi$-component

$$
\begin{aligned}
& S_{N}^{\phi}=A^{2}\left\{-16 \pi N J^{\varphi}-2 K^{\varphi r} N_{, r}-2 K^{\varphi \theta} N_{, \theta}-2 m^{r} m^{\theta} N_{, r \theta}^{\varphi}-2 \frac{N_{, r}^{r}}{M}\left[m^{r}\left(\mu_{, r}-\alpha_{, r}\right)-m^{\theta} \alpha_{, \theta}-\frac{M^{r}, r}{M}\right]\right. \\
& -\frac{N_{, \theta}^{r}}{M}\left[2 m^{\theta} \mu_{, r}-\frac{1}{M}\left(M_{, r}^{\theta}+\frac{M_{, \theta}^{r}}{r^{2}}\right)\right]-\frac{N_{, r}^{\theta}}{M}\left[2 m^{r} \mu_{, \theta}-\frac{1}{M}\left(r^{2} M_{, r}^{\theta}+M_{, \theta}^{r}\right)\right]-2 \frac{N^{\theta}, \theta}{M}\left[m^{\theta}\left(\mu_{, \theta}-\alpha_{, \theta}\right)\right. \\
& \left.-m^{r}\left(\alpha_{, r}+\frac{1}{r}\right)-\frac{M_{, \theta}^{\theta}}{M}\right]-N_{, r}^{\varphi}\left[-m^{r} m^{\theta} \mu_{, \theta}+4 m^{r}\left(m^{r} \alpha_{, r}+m^{\theta} \alpha_{, \theta}\right)+\frac{m^{r}}{M}\left(4 M_{, r}^{r}+M_{, \theta}^{\theta}\right)\right. \\
& \left.+\frac{m^{\theta}}{M}\left(2 M_{, \theta}^{r}+r^{2} M_{, r}^{\theta}\right)\right]-N_{, \theta}^{\varphi}\left[-m^{r} m^{\theta} \mu_{, r}+4 m^{\theta}\left(m^{r} \alpha_{, r}+m^{\theta} \alpha_{, \theta}\right)+\frac{3}{r} m^{r} m^{\theta}+\frac{m^{r}}{M}\left(\frac{M^{r}, \theta}{r^{2}}+2 M_{, r}^{\theta}\right)\right. \\
& \left.+\frac{m^{\theta}}{M}\left(M_{, r}^{r}+4 M_{, \theta}^{\theta}\right)\right]-\frac{N^{r}}{M}\left\{m^{r}\left[2 \frac{\mu_{, r}}{r}-4 \frac{\alpha_{, r}}{r}-\frac{1}{r^{2}}-\left(\mu_{, r}\right)^{2}-2\left(\alpha_{, r}\right)^{2}+4 \alpha_{, r} \mu_{, r}+\frac{M_{, r r}}{M}-2 \frac{A_{, r r}}{A}\right]\right. \\
& +m^{\theta}\left(-\mu_{, r} \mu_{, \theta}+4 \alpha_{, \theta} \mu_{, r}-2 \frac{\alpha_{, \theta}}{r}-2 \alpha_{, r} \alpha_{, \theta}+\frac{M_{, r \theta}}{M}-2 \frac{A_{, r \theta}}{A}\right)+\frac{1}{M}\left[M_{, r}^{r}\left(\mu_{, r}-4 \alpha_{, r}-\frac{1}{r}\right)-M_{, r}^{\theta}\left(\mu_{, \theta}\right.\right. \\
& \left.\left.\left.+2 \alpha_{, \theta}\right)+2 M_{, \theta}^{\theta}\left(\mu_{, r}-\alpha_{, r}-\frac{1}{r}\right)-M_{, r r}^{r}-M_{, r \theta}^{\theta}\right]\right\}-\frac{N^{\theta}}{M}\left\{m ^ { r } \left[-\mu_{, r} \mu_{, \theta}+\left(4 \alpha_{, r}+\frac{2}{r}\right) \mu_{, \theta}-2 \frac{\alpha_{, \theta}}{r}\right.\right.
\end{aligned}
$$




$$
\begin{aligned}
& \left.-2 \alpha_{, r} \alpha_{, \theta}+\frac{M_{, r \theta}}{M}-2 \frac{A_{, r \theta}}{A}\right]+m^{\theta}\left[-\left(\mu_{, \theta}\right)^{2}-2\left(\alpha_{, \theta}\right)^{2}+4 \alpha_{, \theta} \mu_{, \theta}+\frac{M_{, \theta \theta}}{M}-2 \frac{A, \theta \theta}{A}\right]+\frac{1}{M}\left[2 M_{, r}^{r}\left(\mu_{, \theta}-\alpha_{, \theta}\right)\right. \\
& \left.\left.-M_{, \theta}^{r}\left(\mu_{, r}+2 \alpha_{, r}+\frac{1}{r}\right)+M_{, \theta}^{\theta}\left(\mu_{, \theta}-4 \alpha_{, \theta}\right)-M_{, r \theta}^{r}-M_{, \theta \theta}^{\theta}\right]\right\}-\left(m^{r}\right)^{2} N_{, r r}^{\varphi}-\left[\frac{3}{A^{2}}-\left(m^{r}\right)^{2}\right] \beta_{, r} N_{, r}^{\varphi} \\
& \left.-\left(m^{\theta}\right)^{2} N_{, \theta \theta}^{\varphi}-\left[\frac{3}{(r A)^{2}}-\left(m^{\theta}\right)^{2}\right] \beta_{, \theta} N_{, \theta}^{\varphi}+\left(m^{\theta}\right)^{2} \cot \theta N_{, \theta}^{\varphi}\right\}
\end{aligned}
$$

[1] N. Stergioulas and J. L. Friedman, ApJ 444, 306 (1995).

[2] T. Nozawa, N. Stergioulas, E. Gourgoulhon, and Y. Eriguchi, A\&AS 132, 431 (1998).

[3] Y. Eriguchi, E. Müller, and I. Hachisu, A\&A 168, 130 (1986).

[4] K. Ioka and M. Sasaki, ApJ 600, 296 (2004).

[5] H. Komatsu, Y. Eriguchi, and I. Hachisu, MNRAS 237, 355 (1989).

[6] E. Gourgoulhon and S. Bonazzola, Phys. Rev. D 48, 2635 (1993).

[7] C. W. Misner, K. S. Thorne, and J. A. Wheeler, Gravitation (San Francisco: W.H. Freeman and Co., 1973).
[8] N. Stergioulas, T. A. Apostolatos, and J. A. Font, MNRAS 352, 1089 (2004).

[9] I. W. Roxburgh, Ap\&SS 27, 425 (1974).

[10] S. Bonazzola, E. Gourgoulhon, M. Salgado, and J. A. Marck, A\&A 278, 421 (1993).

[11] G. B. Cook, S. L. Shapiro, and S. A. Teukolsky, ApJ 398, 203 (1992).

[12] F. H. Seguin, ApJ 197, 745 (1975).

[13] R. A. D'Inverno, Introducing Einstein's relativity (New York: Oxford University Press, 1992). 